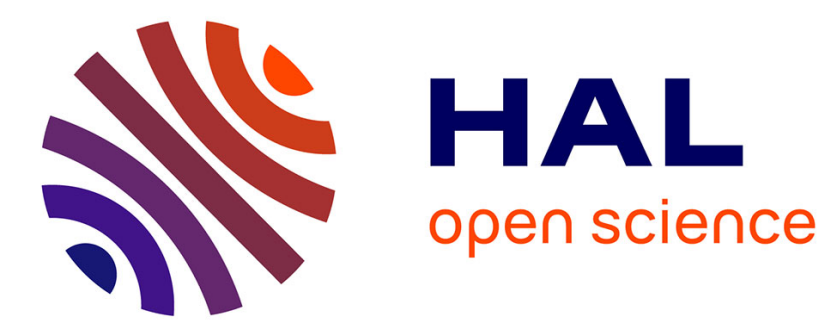

\title{
Patrimoine privé et retraite en France
}

Thomas Blanchet, Yves Dubois, Anthony Marino, Muriel Roger

\section{To cite this version:}

Thomas Blanchet, Yves Dubois, Anthony Marino, Muriel Roger. Patrimoine privé et retraite en

France. 2016. halshs-01292412

\section{HAL Id: halshs-01292412 \\ https://shs.hal.science/halshs-01292412}

Preprint submitted on 23 Mar 2016

HAL is a multi-disciplinary open access archive for the deposit and dissemination of scientific research documents, whether they are published or not. The documents may come from teaching and research institutions in France or abroad, or from public or private research centers.
L'archive ouverte pluridisciplinaire HAL, est destinée au dépôt et à la diffusion de documents scientifiques de niveau recherche, publiés ou non, émanant des établissements d'enseignement et de recherche français ou étrangers, des laboratoires publics ou privés. 


\title{
PARISSCHOOL OF ECONOMICS
}

WORKING PAPER N $2016-03$

Patrimoine privé et retraite en France

\author{
Thomas Blanchet \\ Yves Dubois \\ Anthony Marino \\ Muriel Roger
}

JEL Codes: D31, D91, H55

Keywords: Patrimoine, Retraites, Cycle de vie

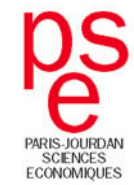




\title{
Patrimoine privé et retraite en France ${ }^{1}$
}

\author{
Thomas Blanchet (ENSAE-PSE) \\ Yves Dubois (INSEE) \\ Anthony Marino (INSEE) \\ Muriel Roger (PSE-INRA, Banque de France)
}

Février 2016

Résumé :

L'existence d'un système de retraite modifie les comportements d'épargne des ménages. Nous estimons le phénomène de substitution entre épargne privée et montant des droits à pension dans le cas français à partir des données de l'enquête Patrimoine et du modèle de microsimulation Destinie de I'INSEE. Les comportements d'épargne sont modélisés à l'aide du modèle de Gale (1998). Les résultats mettent en évidence des comportements de substitution entre l'épargne des ménages et leurs droits à pension. L'amplitude de ces effets est sensible aux paramètres d'actualisation des agents. Une hausse des pensions publiques induit une diminution de l'épargne complémentaire retraite et une diminution de l'investissement immobilier. Ce second effet est prédominant.

Classification JEL : D31, D91, H55

Mots clés : patrimoine, retraites, cycle de vie

Abstract:

The existence of a pension system affects the saving behavior of households. We estimate the substitution effect between private savings and pension wealth using the French wealth survey Patrimoine and the pension microsimulation model Destinie of the French NIS (INSEE). Saving behavior is modeled after Gale (1998). Our results highlight the existence of a substitution effect between household savings and their pension. The magnitude of those effects is sensitive to the discount rate of agents. An increase in public pensions leads to a decrease in complementary pension products and real estate investment. The effect of the latter dominates.

JEL Classification: D31, D91, H55

Keywords: Wealth, Social Security, Pensions, Life Cycle

\footnotetext{
${ }^{1}$ Nous remercions Antoine Bozio, Malik Koubi, Corinne Prost, un referee anonyme ainsi que les participants à l'École thématique Évaluation des Politiques Publiques (TEPP-CNRS) et au séminaire du Département des études économiques (INSEE) pour leurs commentaires. Les erreurs et omissions qui pourraient subsister sont de notre responsabilité. Cet article ne reflète pas la position de l'Insee ni de la Banque de France et n'engage que ses auteurs.
} 


\section{Introduction}

Les systèmes de retraite ont des effets complexes sur les comportements d'accumulation des ménages. Le modèle standard de cycle de vie (Modigliani et Brumberg, 1954) prédit, en l'absence de pensions, une période d'accumulation en début de vie afin d'obtenir un capital permettant de lisser la consommation et d'assurer la subsistance après la cessation d'activité. Les systèmes de retraite, en assurant un revenu en fin de vie, viennent modifier les comportements d'épargne. L'objectif de cet article est d'analyser les liens entre les comportements d'accumulation des ménages français durant leur période d'activité et leur future pension de retraite. Pour cela, nous utilisons conjointement les données de l'enquête Patrimoine, qui fournissent des informations très précises sur le patrimoine des ménages, et le modèle de micro-simulation Destinie ${ }^{2}$ qui permet d'imputer les droits à pension.

Au niveau théorique, la mise en place d'un système de pension a des effets contradictoires sur l'épargne des ménages : une réduction de l'épargne hors retraite, car les droits à retraite se substituent aux autres actifs; un accroissement de l'épargne car, en permettant une cessation d'activité plus précoce, les systèmes de retraite induisent une demande plus grande de financement. De plus, l'équivalent patrimonial des droits à pension, défini comme la somme actualisée des pensions de retraite, est un actif atypique. C'est un actif non liquide, ce qui peut induire une épargne plus élevée que nécessaire en début de vie quand les ménages font face à des contraintes de liquidités. Mais c'est aussi une assurance contre le risque de longévité, ce qui peut réduire l'épargne privée.

La majorité des travaux empiriques sur les liens entre système de retraite et épargne se sont concentrés sur la mesure de l'effet d'éviction des systèmes de pension sur l'accumulation privée. Ils ont conduit à des conclusions variées. L'utilisation de sources de données, de modélisations et de stratégies d'identification différentes amènent à des estimations allant d'un léger effet positif des pensions de retraite sur l'accumulation des ménages à une substitution totale. Les estimations sur données microéconomiques de Kotlikoff (1979) concluent à un taux de substitution avec la richesse nette supérieur à $60 \%$ pour un indicateur des droits à pension défini comme la valeur actualisée individuelle des cotisations payées par le ménage à la date des estimations. En estimant un modèle classique d'équation de patrimoine dans lequel l'indicateur de la richesse retraite est défini par la valeur actualisée des droits à pension, King et Dicks-Mireaux (1983) sur données canadiennes et Hubbard (1986) sur données américaines obtiennent des valeurs 3 fois plus faibles. Gale (1998) a mis en évidence l'importance de considérer, dans les estimations empiriques, l'horizon de planification d'individus d'âges différents pour mesurer les effets de substitution. Il déduit d'un modèle simple de cycle de vie un facteur $Q$, permettant d'ajuster l'indicateur de richesse retraite en fonction de l'âge observé et de l'âge de départ à la retraite dans les estimations. Gale (1998), sur les données américaines du SCF, conclut que cet ajustement conduit à des niveaux de substitution plus élevés que ceux obtenus dans les estimations précédentes. Les coefficients obtenus sont parfois non significativement différents d'un cas de substitution parfaite. L'ajustement de Gale est devenu standard dans la littérature. Hurd et al. (2009) estiment des effets de substitution compris entre 23

\footnotetext{
${ }^{2}$ Le modèle de micro-simulation Destinie, développé par l'INSEE depuis le milieu des années 1990, permet de simuler des montants de pension de retraite pour les individus en fonction des caractéristiques de leur carrière passée et de la législation à laquelle ils sont soumis. Les composantes futures des carrières sont simulées en tenant compte des caractéristiques individuelles.
} 
et $44 \%$ sur les données européennes du Health and Retirement Study couplées avec les données de I'English Longitudinal Study of Ageing et les modèles de pension de I'OECD. Avec les données SHARELIFE, Alessie, et al. (2013) obtiennent un effet de substitution compris entre 17 et 30\%. En utilisant une méthode par variables instrumentales Engelhardt \& Kumar (2011) estiment, sur données américaines, des effets de substitution de l'ordre de 53 à $67 \%$. Bien que les régressions effectuées en intégrant la correction de Gale puissent tendre à sur-corriger le biais et ainsi à surestimer l'effet d'éviction (Blau 2011), la méthode fournit un cadre pertinent qui permet une comparaison des estimations des effets de substitution.

Selon les articles, les estimations sont menées à partir de différents indicateurs des droits à pension : observés, simulés ou issus des anticipations des agents. Les travaux de Chan et Stevens (2008) ont montré que si les individus réagissent aux incitations des systèmes de retraite, ils modifient leur comportement, non pas nécessairement en fonction des paramètres des systèmes, mais en fonction de la connaissance qu'ils en ont. Dans ce cadre, la littérature récente sur les comportements de départ à la retraite privilégie des variables d'anticipations des agents sur leur taux de remplacement ou leur niveau de retraite future pour expliquer les choix de cessation d'activité en fin de carrière. Néanmoins, si l'information des ménages sur les systèmes de retraite est bonne, les variables de niveaux de pension observées, simulées ou anticipées coïncident. Il est donc important de mesurer le niveau de connaissance des populations sur leur système de retraite ${ }^{3}$. Celui-ci est hétérogène selon les pays et les groupes de populations comme l'illustrent Gustman et Steinmeier (2004, 2005). Pour la France, Arrondel, Masson et Soulat (2013) montrent que la majorité de la population a une bonne connaissance du système, en particulier des caractéristiques principales utilisées dans le modèle de microsimulation Destinie. Les estimations seront donc réalisées en utilisant pour les droits à pension des valeurs prédites à partir de ce modèle, les anticipations des agents sur leurs droits futurs n'étant pas connues.

Nous nous plaçons dans un cadre d'étude analogue à celui utilisé dans les travaux de recherche récents. Comme Gale (1998), nous considérons un modèle de cycle de vie dans lequel le ménage choisit, à la date initiale, sa séquence de consommation afin de maximiser son utilité intertemporelle sous sa contrainte budgétaire. II en découle un modèle empirique permettant d'estimer l'ajustement de la richesse nette aux droits à pension. L'hétérogénéité des préférences des ménages vis-à-vis de l'épargne peut biaiser les estimations. Pour pallier à ce problème, nous utilisons une méthode par variables instrumentales. Afin de tester la robustesse de nos résultats, nous effectuons nos estimations en faisant varier les valeurs des paramètres du modèle sous-jacent. Nous mettons ainsi en évidence la sensibilité des résultats à la valeur des paramètres théoriques et donc la difficulté intrinsèque de mesurer l'effet d'éviction. Nous développons deux pistes complémentaires afin de compléter l'analyse. Tout d'abord, la myopie des agents étant une des justifications à l'existence d'un système de retraite obligatoire, les modèles d'accumulation de la richesse nette des ménages sont estimés sur des sous-groupes présentant des niveaux de préférence pour le présent différents. Les estimations ne mettent pas évidence d'effets différenciés pour les sous-groupes. Nous décomposons ensuite la richesse nette en différents actifs afin d'identifier pour quel type de patrimoine se fait la substitution avec la retraite. Celle-ci a lieu principalement avec le patrimoine

\footnotetext{
${ }^{3}$ Des dispositifs d'information sur les droits à pension futurs (simulateurs proposés par les caisses de retraite, envoi de relevés de situation) ont été mis en place récemment dans de nombreux pays pour améliorer l'information des affiliés. C'est le cas par exemple du GIP info-retraite (http://www.info-retraite.fr/) créé en France suite à la loi du 21 août 2003.
} 
immobilier.

La seconde partie de l'article est consacrée à la présentation du modèle empirique, la troisième à la présentation des données et du simulateur de pension. La quatrième présente les résultats des estimations.

\section{Modèle Empirique}

L'article de Gale (1998) a mis en évidence l'importance d'ajuster la valeur de la pension à l'âge lorsque l'on cherche à estimer l'effet d'éviction de la retraite sur le patrimoine des ménages. La spécification du coefficient de correction ( $Q$ de Gale) repose sur une modèle de cycle de vie simple dont les hypothèses peuvent paraître trop contraintes (substitution parfaite). L'auteur argumente toutefois que le coefficient de correction peut s'appliquer dans un cas de substitution imparfaite.

Les articles récents (Attanasio et Brugiavini 2003, Attanasio et Rohwedder 2003, Engelhardt et Kumar 2011, Alessie, Angelini et van Santen 2013) reprennent le modèle de Gale. Le modèle théorique simple sert de base aux interprétations du modèle empirique. Toutefois, les modèles estimés sont plus flexibles que la forme stricte découlant du modèle théorique simple. En effet, celuici ne modélise pas, par exemple, les comportements d'épargne pour des motifs de précaution ou de transmission, l'introduction de ces éléments dans le modèle théorique, en complexifiant la résolution, ne débouche pas sur des solutions analytiques simples.

Nous nous plaçons donc dans un cadre d'étude analogue à celui utilisé dans les travaux de recherche récents. Comme Gale (1998), nous considérons un modèle dynamique standard du consommateur dans lequel le ménage choisit à la date initiale sa séquence de consommation afin de maximiser son utilité intertemporelle sous sa contrainte budgétaire. Le programme du consommateur est donné par :

$$
\begin{gathered}
\max _{\left\{C_{t}\right\}} V=\int_{0}^{t} \frac{C_{t}^{1-\rho}}{1-\rho} e^{-\delta t} d t \\
\text { sous la contrainte } \int_{0}^{T} C_{t} e^{-r t} d t \leq \int_{0}^{R} E_{t} e^{-r t} d t+\int_{R}^{T} B_{t} e^{-r t} d t
\end{gathered}
$$

Avec une fonction d'utilité instantanée iso-élastique, $t$ un indice de temps ou d'âge, $C$ le niveau de consommation, $\rho$ le coefficient d'aversion relative au risque, $\delta$ la préférence pour le présent, $E_{t}$ le revenu au cours de la vie active, $r$ le taux d'intérêt supposé fixe dans le temps, $B_{t}$ le niveau de pension, $\mathrm{R}$ l'âge optimal de départ à la retraite, et $\mathrm{T}$ la durée de vie.

La résolution du programme implique :

$$
C_{t}=C_{0} e^{\left(\frac{r-\delta}{\rho}\right) t}
$$

avec 


$$
\begin{gathered}
C_{0}=\frac{x}{e^{x T}-1}\left(\int_{0}^{R} E_{t} e^{-r t} d t+\int_{R}^{T} B_{t} e^{-r t} d t\right) \\
x=\frac{r-\delta}{\rho}-r
\end{gathered}
$$

Pour $\mathrm{A}<\mathrm{R}$, La richesse hors retraite du ménage à l'âge ${ }^{4} \mathrm{~A}, W_{A}$, correspond à la valeur actualisée de l'épargne passée. Elle est donnée par :

$$
W_{A}=\int_{0}^{T}\left(E_{t}-C_{t}\right) e^{r(A-t)} d t
$$

Si l'on substitue (1) dans (2) on obtient :

$$
W_{A}=\int_{0}^{A} E_{t} e^{r(A-t)} d t-Q\left[\int_{0}^{R} E_{t} e^{r(A-t)} d t\right]-Q\left[\int_{R}^{T} B_{t} e^{r(A-t)} d t\right]
$$

avec, pour S correspondant à la durée de cotisation à l'âge $A$,

$$
Q=\left\{\frac{e^{x S}-1}{e^{x T}-1}\right.
$$

Dans le modèle de base présenté ci-dessus, la substitution entre épargne privée et retraite est considérée comme parfaite. Or, l'existence, entre autres, de contraintes de liquidités en début de vie active ou l'impossibilité d'emprunter de façon anticipée ses revenus de retraite qui rendent cette épargne illiquide impliquent une substitution imparfaite entre les deux types de patrimoine. Dans ce cas, pour une augmentation de l'équivalent patrimonial des droits à pension d'une unité, seule une fraction est allouée à la consommation, le reste étant soit consommé après le départ à la retraite, soit transmise aux héritiers. Gale (1998) montre que la correction proposée dans le cadre du modèle avec substitution parfaite reste valide.

Le modèle illustre l'importance, dans l'étude de la substitution entre épargne privée et épargne retraite, des différences d'horizon de planification en fonction de l'âge au sein des ménages et de la prise en compte des revenus sur l'ensemble du cycle de vie.

L'implémentation empirique du modèle théorique est donnée par :

$$
W_{i}=\beta_{0}+\beta_{1} Y_{i}+\beta_{2} P_{i}+\gamma X_{i}+u_{i}
$$

avec i l'indice du ménage, $Y_{i}$ la variable de revenu du ménage, $P_{i}$ la variable de pension du ménage, $X_{i}$ un vecteur de caractéristiques sociodémographiques et $u_{i}$ le terme d'erreur.

4 L'âge considéré est l'âge de la personne de référence du ménage. 
On définit $P_{i}$ comme la somme actualisée des pensions du ménage. Dans les estimations empiriques, les valeurs retenues pour cet indicateur seront successivement l'indicateur brut puis la valeur ajustée au moyen du coefficient $Q$ de Gale. Nous ferons varier les valeurs de $x$ et $r$ afin de tester la robustesse des estimations au choix des paramètres du modèle théorique sous-jacent.

On définit $Y_{i}$ comme la variable de revenus du ménage. Nous estimons les modèles empiriques en considérant la spécification proposée par Alessie et al (2013)

$$
Y_{i}=\int_{0}^{A} E_{t} e^{-r(A-t)} d t-Q\left[\int_{0}^{R} E_{t} e^{-r(A-t)} d t\right]
$$

Plusieurs problèmes se posent lors de l'estimation OLS du modèle empirique qui sont dus aux erreurs de mesure sur le montant de la pension et à l'hétérogénéité individuelle dans les préférences des ménages vis-à-vis de l'épargne.

Pour pallier au biais dans les estimations que peuvent induire ces différents éléments, nous utilisons une méthode par variables instrumentales. L'idée, analogue à celle de Engelhardt et Kumar (2011) est de construire un instrument pour une valeur moyenne des carrières des individus et non des carrières individuelles. Dans ce cas, la variabilité issue de l'instrument est due uniquement aux spécificités (non-linéarités) des modèles de pensions et non aux différences, potentiellement endogènes, des carrières des individus.

\section{Données et simulateur de pensions}

\section{L'enquête Patrimoine}

Les données sont issues de l'enquête Patrimoine 2009-2010 de l'Insee. 15000 ménages ont été interrogés entre octobre 2009 et janvier 2010 sur le montant et la composition de leur patrimoine. L'enquête contient de plus un module spécifique, dont les questions ont été posées à $1 / 3$ des ménages, afin de connaître leur aversion pour le risque et leur préférence pour le présent. Nous avons restreint notre analyse aux ménages qui ne comportent pas d'indépendants, le modèle de simulation des pensions étant moins précis pour cette sous-population. Nous avons de plus exclu les ménages dont la personne de référence est âgée de plus de 60 ans car le modèle de Gale (1998) ne s'applique qu'aux individus durant leur vie active. L'échantillon total est composé de 5752 ménages, le sous-échantillon risque de 1887 ménages.

\section{Variables dépendantes}

La variable dépendante principale est le patrimoine net du ménage. II est défini comme la somme des actifs financiers et des actifs réels, immobiliers et professionnels, moins l'endettement du ménage. Le recensement des différents actifs et passifs est très précis dans l'enquête Patrimoine. Le patrimoine financier regroupe l'ensemble des montants détenus par les ménages sur les comptes 
de dépôts - comptes courants, livrets d'épargne, les fonds communs de placements, les obligations et actions détenues directement par le ménage, les actifs détenus dans le cadre de régimes de retraite privés volontaires, les assurances-vie avec couverture du risque de décès, et les autres actifs financiers complexes. Nous avons défini 3 variables supplémentaires détaillant des composantes spécifiques du patrimoine financier : l'épargne retraite, l'assurance vie et les valeurs mobilières. Le patrimoine immobilier est défini comme la valeur estimée à la revente par le ménage de tous ses biens immobiliers, résidence principale, résidences secondaires ou biens immobiliers de rapport. Nous avons défini une variable supplémentaire détaillant une composante spécifique : les autres biens immobiliers. Le patrimoine professionnel est défini de manière analogue comme la valeur estimée à la revente de tous les biens à usage professionnel détenus par le ménage ${ }^{5}$. L'enquête fournit de plus la somme des montants d'endettement privé ou professionnel, ce qui permet le calcul du patrimoine net. Tous les actifs des ménages ne présentent pas les mêmes caractéristiques, ils peuvent être plus ou moins liquides par exemple ce qui peut induire des mécanismes de substitution différenciés entre épargne privée et équivalents patrimoniaux des droits à pension. Les modèles seront donc estimés successivement sur le patrimoine net du ménage puis en prenant comme variables dépendantes le patrimoine financier et le patrimoine immobilier et leurs différentes composantes. Les montants des différentes composantes du patrimoine total sont des montants bruts, l'endettement des ménages n'ayant pas été réaffecté poste par poste.

(Insérer tableaux 1 et 2)

Les tableaux 1et 2 présentent les distributions de patrimoines pour l'ensemble de notre échantillon et pour le sous-échantillon « risque ». On constate que les distributions sont analogues.

Les montants de patrimoines de nos échantillons sont moins élevés que ceux de la population générale puisque le patrimoine brut médian en France en 2010 est estimé à $150200 €$ (Hélène Chaput, Kim-Hoa Luu Kim, Laurianne Salembier et Julie Solard 2011). Ces différences s'expliquent par la sélection des ménages hors indépendants dont le chef de ménage est âgé de moins de 60 ans. Les deux catégories de population exclues, indépendants et retraités, sont, pour des raisons qui diffèrent, celles qui possèdent en moyenne les plus hauts niveaux de patrimoine (Bourdieu, Rapoport et Roger 2014).

On retrouve dans le tableau 1 le résultat standard sur la forte concentration de la richesse avec une détention de patrimoine très faible dans le premier quartile et jusqu'à la médiane pour le patrimoine financier. La forte croissance du patrimoine total entre le premier quartile et la médiane s'explique par la détention de patrimoine immobilier. De façon standard, on observe une diversification de la structure du patrimoine lorsque son montant augmente (Arrondel, Roger et Savignac 2013). Le patrimoine des ménages du premier décile est composé quasi exclusivement d'actifs financiers et de biens durables. Lorsque le patrimoine brut total s'accroît, la part du patrimoine financier et des autres biens diminue au profit de celle du patrimoine immobilier qui

\footnotetext{
5 L'échantillon sélectionné étant un échantillon de ménages ne comportant pas de travailleurs indépendants, cette composante est marginale.
} 
augmente. On observe ensuite un basculement à partir du troisième quartile avec un accroissement très net de la contribution du patrimoine professionnel et des autres biens immobiliers à la valeur totale du patrimoine des ménages.

\section{Variables sociodémographiques et préférences des ménages}

L'ensemble des caractéristiques sociodémographiques des ménages de l'échantillon est donné en annexe 1 . Nous ne présentons ici que celles qui ont une influence spécifique pour l'estimation des effets de substitution entre épargne privée et équivalent patrimonial des droits à pension.

L'âge est une variable explicative fondamentale des comportements d'accumulation des ménages dans les modèles à cycle de vie. Les ménages accumulent du patrimoine au cours de leur vie active par prévoyance (financement de la retraite, de la dépendance et pour faire face aux aléas de la vie, chômage, santé, etc.), voire pour transmettre un patrimoine à leurs descendants. Dans cette évolution du patrimoine global du ménage selon l'âge, l'immobilier joue un rôle marquant, avec des comportements fréquents d'achats de logements en début de cycle de vie. La distribution des âges des personnes de référence dans notre échantillon est donnée Graphique 1. Elle est concentrée vers les 40-60 ans, à l'image de la population française

Nous n'avons qu'une faible proportion de ménages qui déclarent avoir reçu un héritage ou une donation (tableau 3). Compte tenu de l'allongement de la durée de vie, l'âge moyen au moment de l'héritage est de 50 ans ce qui peut expliquer ce phénomène. Il est néanmoins important de tenir compte de l'existence d'un héritage ou d'une donation reçue au sein du ménage dans nos estimations. En effet, lorsque l'on s'intéresse au patrimoine des ménages, c'est un élément explicatif extrêmement discriminant. Comme le montrent Garbinti, Lamarche et Salambier (2012), le patrimoine médian des héritiers est plus élevé que celui des autres ménages.

(Insérer tableau 3)

La variable de préférences pour le présent (tableau 3) est directement issue des travaux d'Arrondel et al. (2004). Dans l'enquête Patrimoine 2009-2010, un tiers des ménages de l'échantillon ont été interrogées sur la manière dont ils évaluent leur rapport au risque. Les questions ont été posées à une personne par ménage, principalement le chef de ménage ou son conjoint. Comme détaillé dans Arrondel et Calvo (2009), les individus étaient invités à se placer sur une échelle de préférence allant de 0 à $10^{6}$. On observe une forte masse en 5 , point modal de la distribution et une

\footnotetext{
${ }^{6}$ La question posée est la suivante. En matière d'attitude à l'égard du FUTUR, essayez de vous situer sur l'échelle : Entre 0 ( personne vivant au jour le jour et prenant la vie comme elle vient, sans trop songer au lendemain ni se projeter dans l'avenir) et 10 (personne préoccupée par son avenir ayant des idées bien arrêtées sur ce qu'elle voudrait être ou faire plus tard).
} 
légère concentration sur les valeurs supérieures, qui correspondent à une préférence pour le présent plus faible. Dans la suite, nous travaillerons avec une variable en 3 classes :

- $\quad 1$ : forte préférence pour le présent regroupant les valeurs strictement inférieures à 5 ;

- 2 : situation médiane correspondant à la valeur 5 ;

- 3: préoccupation plus importante pour l'avenir regroupant les valeurs strictement supérieures à 5 .

\section{Distribution du Q de Gale}

Le $Q$ de Gale est une fonction du coefficient d'aversion relative au risque, de la préférence pour le présent, du taux d'intérêt, de la durée de cotisation au moment où l'on observe le ménage et de la durée de vie. Par la suite, nous travaillerons sous une hypothèse d'égalité de la préférence pour le présent au taux d'intérêt. On obtient :

$$
Q=\left\{\frac{e^{-r S}-1}{e^{-r T}-1}\right.
$$

avec $r$ le taux d'intérêt, $\mathrm{S}$ la durée de cotisation et T la durée de vie.

Le graphique 2 présente les distributions des $Q$ de Gale, pour l'échantillon d'étude et trois valeurs distinctes de $r$ comprises entre 0,01 et 0,03 . Plus la valeur du taux d'intérêt est faible, moins la distribution de $Q$ est concentrée vers 0 et donc plus la correction sera faible puisque le facteur correcteur devant la variable de pension se rapproche de 1.

(Insérer graphique 2)

\section{Simulation des pensions : le modèle Destinie}

Les pensions sont simulées à l'aide du modèle de micro-simulation Destinie, développé par I'INSEE depuis le milieu des années 1990. Les simulations ont été effectuées à l'aide de la version 2 du modèle (Blanchet, Buffeteau, Crenner et Le Minez 2010 ; Bachelet, Leduc et Marino 2014). Ce modèle est constitué de deux blocs séparés: un générateur de biographies démographiques et professionnelles et un simulateur de retraite qui consiste en une bibliothèque d'utilitaires permettant la construction de projections de retraites sur mesure, sur la base des biographies issues de la première étape.

Nous avons simulé les retraites en prenant comme hypothèse un départ à taux plein. Comme nous étudions l'épargne en 2009-2010, les règles appliquées pour le calcul des retraites sont celles de 2009 et l'on considère la structure démographique des ménages telle qu'observée dans l'enquête Patrimoine 2009. Concernant la carrière professionnelle, les hypothèses sont les hypothèses 
traditionnelles du modèle Destinie. Le modèle tire parti du fait que l'enquête Patrimoine fournit des informations sur les carrières jusqu'en 2009. Pour chaque année au-delà de l'âge de sortie du système scolaire, le statut de la personne sur le marché du travail est connu : salarié du secteur public ou privé, travailleur indépendant, chômeur, retraité ou inactif. Toutefois, les carrières salariales passées ne sont pas renseignées dans l'enquête, les séquences de salaire passées sont donc imputées à partir d'équations de salaires. Les variables microéconomiques qui déterminent le salaire sont l'âge de fin d'études, l'ancienneté dans l'emploi, introduite sous forme quadratique, et une composante stochastique incluant un effet fixe individuel et un résidu auto-corrélé. La part déterministe de ces équations est complètement différenciée entre les secteurs public et privé.

Après 2009, toutes ces informations sont projetées. Les transitions entre les états sur le marché du travail se font selon des processus markoviens de premier ordre dans lesquels les probabilités de transition sont estimées à partir des enquêtes emploi conduites par I'INSEE. Ces probabilités dépendent des caractéristiques individuelles. Conditionnellement au statut sur le marché du travail, les salaires annuels sont projetés en recourant aux mêmes équations de salaire que celles qui sont utilisées pour la reconstitution rétrospective des carrières, augmentées d'un terme représentant les progrès de productivité.

Les évolutions des salaires et les risques de chômage sont estimés à partir des données observées. S'il est difficile de considérer que les individus peuvent anticiper de façon parfaite leur trajectoire future, la manière dont les simulations sont faites est cohérente avec l'hypothèse que les agents connaissent les distributions des probabilités d'évolution de carrière ou de chômage, mais non les réalisations individuelles. Les anticipations des agents dépendent ainsi des distributions auxquelles ils font face. Le modèle Destinie prédit les carrières à partir de ces distributions.

Les valeurs des pensions sont simulées en prenant comme hypothèse un départ à taux plein, qui correspond à la valeur modale des choix dans le système français. La connaissance limitée des différents dispositifs alternatifs comme la surcote, la décote ou la retraite progressive (Aubert, Barthélemy et Benalla, 2012, COR 2013) augmente la probabilité de choix de cette modalité de départ. De plus, le taux plein est un des éléments bénéficiant $d$ 'une bonne connaissance de la part des assurés (Bridenne 2006, Aubert, Barthélemy et Benallah 2012, Arrondel, Masson et Soulat 2013).

Les travaux menés concluent majoritairement à une bonne connaissance du système en général, mais à une plus faible connaissance par les individus du détail de leurs droits personnels. Néanmoins, comme souligné par Benallah (2012), la mesure de la connaissance des caractéristiques individuelles des droits à pension est complexe. Les conclusions relatives à un faible niveau d'information sont généralement issues d'une comparaison avec les informations fournies par les administrations en charge des systèmes de retraite. Ces informations peuvent elle-même être incomplètes si certaines bonifications ne sont incluses que lors du calcul des droits à pension au moment de la liquidation ${ }^{7}$. Nous n'avons pas la possibilité d'identifier l'écart entre les valeurs des pensions prédites par le modèle et les anticipations des agents. Les résultats seront donc interprétés en considérant les valeurs prédites pour les pensions comme celles sur lesquelles les ménages basent leur choix d'épargne. Nous discuterons cette hypothèse si nécessaire.

\footnotetext{
${ }^{7}$ C'est par exemple le cas des bonifications pour enfants dans le système français.
} 
Pour chaque personne, la valeur de la retraite à la date de liquidation est calculée à l'aide du simulateur de retraite du modèle de micro-simulation. On définit ensuite la variable pension comme la valeur actualisée de l'ensemble des pensions reçues de la date de départ jusqu'au décès. Les durées de vie sont basées sur le taux de mortalité de la Human Mortality Database. Les taux de mortalité futurs sont prédits sur la base de projections logarithmiques.

Les simulations de carrière et le calcul des pensions sont faits au niveau individuel puis agrégées au niveau du ménage et enfin actualisées en fonction de l'âge de la personne de référence dans l'enquête. Les graphiques 3 et 4 présentent les distributions des indicateurs de salaires et de pension en fonction de la valeur du taux d'intérêt. De façon standard, la dispersion observée sur les revenus est moindre comparée à celle sur les patrimoines.

(Insérer graphiques 3 et 4)

Comme souligné dans la partie II, pour pallier au biais dans les estimations, nous utilisons une méthode par variables instrumentales. L'idée est de construire des instruments qui correspondent à la richesse retraite pour une carrière moyenne par genre, cohorte et âge de fin d'étude. L'objectif est de neutraliser des facteurs d'hétérogénéité individuelle comme, par exemple, une préférence pour le loisir qui induirait simultanément des salaires plus faibles et un départ précoce. Ces deux éléments ont un impact sur les choix d'épargne du ménage. Des salaires plus faibles entraînent un équivalent patrimonial à pension plus faible; un départ précoce augmente les besoins d'épargne pour la période de retraite. La conjonction de ces deux éléments conduit à une surestimation de l'effet d'éviction.

L'objectif de l'instrumentation est d'estimer l'effet d'éviction en utilisant comme source d'identification les caractéristiques des systèmes de retraite et non l'hétérogénéité individuelle. Pour cela, pour le premier instrument, nous fixons les salaires à la valeur moyenne par genre, cohorte et âge de fin d'étude. Pour le second, nous considérons de plus une carrière complète avec comme âge de liquidation l'âge moyen de liquidation de la cohorte.

\section{Résultats}

\section{Patrimoine net}

Afin de connaître la sensibilité des résultats aux spécifications du modèle empirique sousjacent, nous avons effectué les régressions ${ }^{8}$ en faisant varier les spécifications choisies : valeur des différents paramètres et indicateurs de revenu. Les estimations sont réalisées par OLS puis par variables instrumentales ${ }^{9}$. Dans ce cas, la variabilité issue de l'instrument est due uniquement aux

\footnotetext{
${ }^{8}$ Compte tenu de l'échantillonnage de l'enquête Patrimoine, les régressions sont effectuées en tenant compte de poids de sondage.

${ }^{9}$ Les statistiques de Fisher dans les régressions de première étape sont de l'ordre de 25 , ce qui exclut le risque d'instruments faibles.
} 
spécificités (non-linéarités) des modèles de pensions et non aux différences, potentiellement endogènes, des carrières des individus. Les corrélations entre les instruments et la variable de pension sont présentées dans le tableau 4. Les résultats des estimations dans le tableau 5.

(Insérer tableaux 4 et5)

La prise en compte de l'ajustement augmente de façon sensible le coefficient de l'indicateur de la richesse retraite du ménage. Les résultats sont toujours négatifs et significatifs, quels que soient les paramètres choisis, ce qui signifie qu'une augmentation des droits à pension diminue l'accumulation de patrimoine privé des ménages. ${ }^{10}$ Les effets sont robustes à l'instrumentation. Les coefficients sont toutefois plus faibles que pour les estimations OLS, ce qui marque la prise en compte du biais dans les estimations. Les estimations réalisées illustrent la difficulté de donner une valeur de l'effet d'éviction entre épargne privée et système de retraite. En effet, les résultats sont très dépendants des hypothèses sur le taux d'intérêt, facteur clé de l'actualisation intertemporelle au niveau théorique, mais aussi comme variable d'intérêt dans les décisions intertemporelles des ménages. Compte tenu de cet élément, tous les résultats seront donnés par la suite en fonction des trois valeurs de $r$ retenues pour la description du $Q$ de Gale et qui correspondent à des valeurs admissibles pour le taux d'intérêt à long terme. Dans la présentation des résultats, on s'intéresse plus aux effets qualitatifs et donc aux tendances qu'aux niveaux absolus. II faut noter que les estimations sur les pensions non ajustées sont elles aussi sensibles à la valeur du taux d'intérêt, via l'actualisation des salaires passés, mais les différences sont beaucoup plus faibles et souvent non significatives.

\section{Substitution et myopie des agents}

Le tableau 6 présente les résultats obtenus lorsque l'on restreint l'étude au sous-échantillon pour lequel nous disposons d'informations sur les échelles de risque. Les régressions sont effectuées en considérant les 3 classes définies dans le III. : forte préférence pour le présent, situation médiane, préoccupation plus importante pour l'avenir.

\section{(Insérer tableaux6 et 7)}

La myopie, définie comme une très forte préférence pour le présent des agents, est un des éléments importants dans les discussions sur la pertinence de la mise en place ou du maintien d'un système de retraite obligatoire par répartition. Pour Feldstein (1985), si la substitution entre retraite et épargne privée est importante, le résultat illustre une faible myopie des agents et les effets induits sur l'accumulation du capital d'un système de retraite par répartition est dommageable sur la

\footnotetext{
${ }^{10}$ Nous faisons l'hypothèse qu'il n'existe pas une erreur systématique entre les anticipations des ménages et l'indicateur de droits à pension qui pourrait conduire, par exemple, à observer un niveau d'épargne élevé pour des pensions anticipées faibles et des variables prédites des pensions élevées. Dans ce cas, un faible niveau observé de substitution pourrait correspondre à une erreur sur les anticipations des ménages et non une absence d'effet d'éviction.
} 
croissance. Les résultats obtenus dans le tableau 5 mettent en évidence un effet de substitution, potentiellement fort, entre épargne privée et montant de l'équivalent patrimonial à pension, issu, dans le cas français, essentiellement d'un système de retraite par répartition.

Afin de pouvoir faire les comparaisons, nous avons reproduit le tableau 5 sur le souséchantillon des ménages pour lequel les informations sur la préférence pour le présent sont connues, afin de ne pas mêler dans nos interprétations des effets myopie et des effets d'échantillonnage. Les résultats sur l'ensemble du sous-échantillon risque sont quantitativement et qualitativement analogues à ceux obtenus pour l'ensemble de l'échantillon.

Lorsque l'on effectue les régressions pour les différents sous-groupes en fonction de leur degré de préférence pour le présent, les résultats sont moins souvent significatifs, ce qui peut être dû à une plus faible taille des échantillons. Lorsque les coefficients sont significativement différents de zéro, on n'observe pas de différence entre les sous-groupes composés de personnes ayant les plus fortes et les plus faibles niveaux de préférence pour le présent. La substitution existe, dans des proportions analogues, dans les deux cas. La variable de préférence pour le présent ne semble pas être le facteur décisif des choix de substitution d'épargne. Afin de mieux identifier le phénomène de substitution nous désagrégeons le patrimoine total en fonction de ses composantes principales.

\section{Décomposition des effets de substitution en fonction des composantes du patrimoine}

L'analyse des effets de substitution selon le type de patrimoine est effectuée pour le patrimoine immobilier, le patrimoine financier, ainsi que pour trois composantes particulières du patrimoine financier : l'épargne retraite, l'assurance vie et les valeurs mobilières, et une composante particulière du patrimoine immobilier : les biens immobiliers autres que la résidence principale. Les ménages de notre échantillon ne détiennent pas tous du patrimoine immobilier ou les différentes composantes particulières du patrimoine financier ou immobilier que nous avons considérées. Afin de tenir compte de la censure des distributions, pour les variables concernées, les estimations sont réalisées à l'aide de modèles tobit ou de modèles tobit instrumentés. Les coefficients estimés, pour un taux d'intérêt égal à 0.03 , sont donnés dans le tableau 8. Le tableau 9 fournit les taux de détention prédits par les modèles tobit ainsi que les effets marginaux des indicateurs de pension pour les différentes composantes du patrimoine dont les effets sont significatifs. Les écart-types sont calculés par la Delta-méthode (Oehlert, 1992). Les tableaux correspondants aux deux autres valeurs du taux d'intérêt sont présentés en annexe.

(Insérer tableaux 8 et 9)

Lorsque l'on considère le patrimoine total, l'estimation instrumentée avec ajustement à l'aide du $Q$ de Gale conduit à un niveau élevé de substitution. Le patrimoine financier étant plus liquide, et donc plus à même de s'ajuster en cas de besoin des ménages que le patrimoine immobilier, on pourrait s'attendre à ce que l'effet de substitution provienne de la composante financière de la richesse. L'impact d'une variation du montant de la pension est directement comparable entre le patrimoine total et le patrimoine financier, ces deux grandeurs étant exprimées en niveau et présentes pour tous les ménages. Or les coefficients estimés sont deux fois plus faibles 
pour le patrimoine financier que pour le patrimoine total. Ceci s'explique par un effet de substitution complémentaire qui transite par le canal de l'immobilier.

La décomposition au niveau des différents actifs permet d'affiner l'analyse. Les résultats obtenus pour le patrimoine financier ne signifient pas qu'il n'y ait pas une substitution importante entre des produits financiers spécifiques, dont les supports sont plus proches d'un investissement dans un capital retraite, mais simplement qu'au niveau global, pour l'ensemble des ménages, l'effet de substitution est modéré. De fait, la substitution se fait entre les retraites publiques et les produits d'épargne retraite ${ }^{11}$. Après instrumentation, les coefficients estimés pour les autres produits financiers sont non significatifs. En particulier, l'effet de substitution entre épargne privé et assurance vie. On ne retrouve pas ici le résultat traditionnel selon lequel l'assurance vie serait, en France, un produit financier utilisé par les ménages en vue de préparer leur retraite. Ce dernier élément est en effet mis en avant dans des proportions significatives par les ménages comme motif de détention d'assurance vie (28\% des ménages détenteurs en 2004 selon Darmon Pagenelle 2005). De même, on n'observe pas de substitution directe avec des produits comme les valeurs mobilières dont on peut penser qu'ils correspondent plus à des logiques de placement financier potentiellement à plus court terme que les produits d'assurance-vie ou d'épargne retraite.

Pour le patrimoine immobilier, les effets sont non significatifs lorsqu'on restreint la variable aux seuls biens immobiliers hors résidence principale. La substitution ne se fait donc pas avec les résidences secondaires ou l'immobilier de rapport, mais directement avec la résidence principale. L'effet de l'immobilier est l'effet prédominant dans la substitution. La variation marginale est 10 fois plus élevée pour l'immobilier que pour l'épargne retraite. Comme le montrent Lusardi et Mitchell (2007), dans le cas américain, le patrimoine immobilier est une composante importante des ressources que peuvent mobiliser les ménages pour leur période de retraite. L'existence d'un niveau de pension plus élevé rend moins nécessaire l'assurance d'un revenu potentiel en cas de nécessité que la résidence principale peut représenter. Même si le recours à la richesse immobilière reste faible, Munnell, Soto et Aubry (2007) montrent qu'avec une baisse des pensions, la possibilité d'utiliser sa résidence principale pour en tirer une source de revenu (vente ou viager) semble être une option envisageable pour de plus en plus de ménages âgés.

\section{Conclusion}

L'existence d'un système de retraite a des effets complexes sur les comportements d'accumulation des ménages. Dans cet article, nous avons analysé, pour la France, les effets de substitution entre le montant du patrimoine privé des ménages et la générosité du système de pension en nous plaçant dans un cadre d'étude analogue à celui utilisé dans les travaux de recherches récents. Nous avons introduit dans le modèle empirique, à la suite de Gale (1998), un facteur d'ajustement sur l'indicateur des droits à pension pour tenir compte de l'âge auquel on observe les individus, de leur durée de cotisation à cette date et de leur espérance de vie. De plus, nous avons mobilisé des méthodes d'estimation par variables instrumentales pour tenir compte des biais dans les estimations OLS. Les modèles empiriques sont estimés en utilisant conjointement les données de l'enquête Patrimoine, qui fournissent des informations très précises sur le patrimoine

\footnotetext{
${ }^{11}$ Plan d'épargne retraite populaire (PERP), Retraite surcomplémentaire et Retraite complémentaire volontaire.
} 
des ménages, et le modèle de micro-simulation Destinie, qui permet de prédire les droits à pension. Les résultats sont sensibles aux spécifications choisies. La prise en compte de l'ajustement augmente de façon sensible la substitution estimée entre patrimoine privé et pensions.

Les estimations mettent en évidence un phénomène de substitution entre accumulation privée et richesse retraite. Les résultats illustrent néanmoins la difficulté de donner une valeur de l'effet de substitution car ils sont très dépendants des hypothèses sur le taux d'intérêt, facteur clé de I'actualisation intertemporelle au niveau théorique, mais aussi comme variable d'intérêt dans les décisions intertemporelles des ménages. Compte tenu de cet élément, les résultats sont présentés en tendance et non en niveaux absolus. La myopie des agents étant un élément important dans les discussions sur la pertinence de la mise en place ou du maintien d'un système de retraite obligatoire par répartition nous avons estimé les modèles d'accumulation de la richesse nette des ménages sur des sous-groupes présentant des niveaux de préférence pour le présent différents. Les estimations ne mettent pas évidence d'effets différenciés pour les sous-groupes. Pour finir, nous avons décomposé la richesse nette en différents actifs afin d'identifier pour quel type de patrimoine se font les substitutions entre accumulation privée et pension de retraite. On observe simultanément des phénomènes de substitution au niveau du patrimoine financier et du patrimoine immobilier, avec un effet prédominant de ce dernier actif.

L'importance de l'effet d'éviction pour le patrimoine immobilier, et principalement pour la résidence principale, est un élément dont la compréhension est nécessaire pour expliquer les comportements d'accumulation des ménages car cet actif réel concentre plusieurs qualités. C'est un bien d'usage mais aussi une réserve de valeur (peu liquide) qui peut avoir des propriétés assurantielle pour le financement de la fin de vie et qui est porteur simultanément d'une valeur de transmission. La compréhension de ce phénomène de substitution pourrait être approfondie, en particulier en comparaison internationale dans un cadre où les systèmes de retraite sont hétérogènes. 


\section{Bibliographie}

Alessie, R., Angelini, V. \& van Santen, P., 2013,"Pension Wealth and Household Savings in Europe: Evidence from SHARELIFE",European Economic Review.

Arrondel, L., \& Calvo, H., 2009, "Les Français sont-ils prudents? Patrimoine et risque sur les revenus des méanges",Economie et Statistique, 417-418.

Arrondel, L., A. Masson et L. Soulat (2013), "Les Français et leur retraite : connaissance, inquiétude et attachement", Questions retraite et solidarité - Les études, $\mathrm{n}^{\circ} 2$.

Arrondel, L., Masson, A. \& Verger, D., 2004, "Préférences de l'épargnant et accumulation patrimoniale: les comportements de l'épargnant à l'égard du risque et du temps",Economie et Statistique, 374-375.

Arrondel, L., Roger, M. \& Savignac, F., 2013, "Patrimoine et endettement des ménages dans la zone euro : le rôle prépondérant de l'immobilier", Bulletin de la Banque de France, 192.

Attanasio, O. P. \& Brugiavini, A., 2003,"Social Security and Households Savings"Quarterly Journal of Economics.

Attanasio, O. P. \& Rohwedder, S., 2003, "Pension Wealth and Household Saving: Evidence from Pension Reforms in the United Kingdom," American Economic Review, American Economic Association, 93(5).

Aubert, P., Barthélemy, N., Benallah, S., 2012, "Le départ à la retraite : motivations et connaissance des droits", Dossier Solidarité Santé $n^{\circ} 34$, Drees.

Bachelet, M., Leduc, A. \& Marino, A., 2014, "Les biographies du modèle Destinie II : rebasage et projection", DESE Working Paper, g2014-01, Institut National de la Statistique et des Etudes Economiques.

Benallah, S., 2012, "Le niveau d'information des salariés sur leurs droits à la retraite : mesure et implications", Les cahiers de la Cnav nº 4.

Blanchet, D., Buffeteau, S., Crenner, E. \& Le Minez, S., 2010, "The new Destinie 2 microsimualtion model: main characteristics and illustrative results", DESE Working Paper, g2010-13, Institut National de la Statistique et des Etudes Economiques.

Blau, D., 2011, "Pensions, Household Saving, and Welfare: A Dynamic Analysis," IZA Discussion Papers 5554 , Institute for the Study of Labor (IZA).

Bourdieu, J., Rapoport, B. \& Roger, M., 2014, "Montant et composition du patrimoine des indépendants, avant et après le départ à la retraite ", Economie et Statistique, 472-473.

Bridenne, I., 2006, "Le niveau d'information des assurés en matière de retraite", Dossiers Solidarité et Santé, $n^{\circ} 3$, pp. 51-62. 
Chan,S., Stevens, H.F., 2008, "What You Don't Know Can't Help You: Pension Knowledge and Retirement Decision-Making," The Review of Economics and Statistics, vol. 90(2), pp 253-266.

Chaput, H., Kim, K. H., Salembier, L. \& Solard, J., 2011, "Les inégalités de patrimoine s'accroissent entre 2004 et 2010", INSEE Première $n^{\circ} 1380$, Institut National de la Statistique et des Etudes Economiques.

COR, 2013, "La connaissance de leurs droits par les assurés : synthèse de résultats", http://www.corretraites.fr/IMG/pdf/doc-2031.pdf.

Darmon, C., \& Panegelle, H., 2005, "Patrimoine : quand les ménages prennent de l'assurance", INSEE Première $n^{\circ} 1015$, Institut National de la Statistique et des Etudes Economiques.

Engelhardt, G. V. \& Kumar, A., 2011, "Pensions and Household Wealth Accumulation", Journal of Human Resources

Gale, W. G., 1998, "The Effects of Pensions on Household Wealth: A Reevaluation of Theory and Evidence"Journal of Political Economy.

Gale, W. G. \& Phillips, J. W. R., 2006, "Pensions, Social Security Wealth and Lifetime Earnings: Evidence from the Health and Retirement Study",Center for Retirement Research at Boston College.

Garbinti, B., Lamarche, P., \& Salembier, L., 2012, "Héritages, donations et aides aux ascendants et descendants", Insee-Références Les revenus et le patrimoine des ménages, édition 2012, Institut National de la Statistique et des Etudes Economiques.

Gustman, A., Steinmeier, T., 2004, "What People Don't Know About Their Pensions and Social Security". In William G. Gale, John B. Shoven and Mark J. Warshawsky, editors, Private Pensions and Public Policies, Washington: D.C., Brookings Institution, pp. 57-125.

Gustman, A., Steinmeier, T., 2005, "Imperfect Knowledge of social security and pensions. ", Industrial Relations: A Journal of Economy and Society, 44(2), pp. 373-397.

Hubbard, R. G., 1986, "Pension Wealth and Individual Saving: Some New Evidence", Journal of Money, Credit and Banking, 18 May.pp. 167-178.

Hurd, M., Michaud, P.-C. \& Rohwedder, S., 2009, "The Displacement Effect of Public Pensions on the Accumulation of Financial Assets",Fiscal Studies 33:1, pp. 107-128.

Dicks-Mireaux, L. \& King., M., 1983, "Portfolio Composition and Pension Wealth: An Econometric Study",Financial Aspects of the U.S. Pension System, edited by ZviBodie and John B. Shoven. Chicago: UCP.

Kotlikoff, J. L., 1979, "Testing the Theory of Social Security and Life Cycle Accumulation", American Economic Review, pp. 396-409.

Lusardi, A. \& Mitchell, O., 2007, "Baby Boomer retirement security: The roles of planning, financial literacy, and housing wealth", Journal of Monetary Economics, Volume 54, Issue 1, pp. 205-224. 
Modigliani , F. \& Brumberg, R., 1954, "Utility Analysis and the Consumption Function: An Interpretation of Cross-Section Data", in K. Kurihara, ed., Post Keynesian Economics, Rutgers University Press, New Brunswick.

Munnell, A., Soto, M. \& Aubry, J. P., 2007, "Do people plan to tap their home equity in retirement", Center for Retirement Research, Boston College.

Oehlert, G., 1992, "A note on the Delta Method", The American Statistician, Volume 46, N 1, pp. $27-$ 29. 
Tableau 1 : Distributions du patrimoine des ménages

\begin{tabular}{|l|c|c|c|c|c|}
\hline \hline & Moyenne & Ecart-type & Q1 & Médiane & Q3 \\
\hline \hline Ensemble & & & & & \\
Patrimoine brut & 182 & 263 & 9 & 124 & 270 \\
Patrimoine net & 149 & 240 & 7 & 71 & 220 \\
Patrimoine financier & 30 & 102 & 2 & 8 & 28 \\
$\quad$ épargne retraite* & 9 & 20 & 1 & 3 & 9 \\
assurance vie* & 23 & 74 & 2 & 6 & 19 \\
valeurs mobilières* & 26 & 158 & 1 & 5 & 18 \\
Patrimoine immobilier* & 249 & 183 & 140 & 208 & 303 \\
$\quad$ résidence principale* & 221 & 125 & 142 & 201 & 270 \\
autre immobilier* & 168 & 163 & 74 & 122 & 208 \\
& & & & & \\
Sous-échantillon risque & & & & & \\
Patrimoine brut & 176 & 238 & 9 & 120 & 262 \\
Patrimoine net & 143 & 214 & 7 & 65 & 217 \\
Patrimoine financier & 29 & 69 & 2 & 8 & 29 \\
$\quad$ épargne retraite* & 10 & 21 & 2 & 4 & 9 \\
assurance vie* & 20 & 47 & 2 & 6 & 19 \\
valeurs mobilières* & 23 & 68 & 1 & 4 & 15 \\
Patrimoine immobilier* & 246 & 176 & 140 & 205 & 300 \\
résidence principale* & 218 & 118 & 140 & 199 & 267 \\
autre immobilier* & 170 & 154 & 82 & 122 & 211 \\
\hline \hline Source Enquete Patrimoin
\end{tabular}

Source: Enquête Patrimoine INSEE 2009-2010, 5752 ménages

Note : tous les montants sont en milliers d'euros, ${ }^{*}$ pour les détenteurs 
Tableau 2 : Détentions des différents actifs

\begin{tabular}{|l|c|}
\hline \multicolumn{2}{|c|}{ en \% } \\
\hline \hline Ensemble & Détention \\
Patrimoine financier & 100 \\
$\quad$ épargne retraite & 10,87 \\
assurance vie & 31,03 \\
$\quad$ valeurs mobilières & 17,63 \\
Patrimoine immobilier & 54,98 \\
$\quad$ résidence principale & 50,50 \\
autre immobilier & 15,01 \\
& \\
Sous-échantillon risque & \\
Patrimoine financier & 100 \\
épargne retraite & 10,34 \\
assurance vie & 32,30 \\
$\quad$ valeurs mobilières & 18,39 \\
Patrimoine immobilier & 53,87 \\
résidence principale & 49,99 \\
autre immobilier & 13,75 \\
\hline \hline
\end{tabular}

Source: Enquête Patrimoine INSEE 2009-2010, 5752 ménages 
Tableau 3 : héritage et variable de préférences

\begin{tabular}{|c|c|c|}
\hline & $\begin{array}{c}\text { Fréquenc } \\
\mathrm{e}\end{array}$ & $\begin{array}{c}\text { Pourcentag } \\
\text { e }\end{array}$ \\
\hline $\begin{array}{l}\text { A reçu un héritage ou une donation } \\
\text { (sous échantillon risque) }\end{array}$ & $\begin{array}{l}250 \\
(87)\end{array}$ & $\begin{array}{l}4.35 \\
(4.61)\end{array}$ \\
\hline Echelle de risque : à l'égard du futur & & \\
\hline 0 & 106 & 5.62 \\
\hline 1 & 34 & 1.80 \\
\hline 2 & 56 & 2.97 \\
\hline 3 & 100 & 5.30 \\
\hline 4 & 103 & 5.46 \\
\hline 5 & 595 & 31.53 \\
\hline 6 & 150 & 7.95 \\
\hline 7 & 216 & 11.45 \\
\hline 8 & 254 & 13.46 \\
\hline 9 & 65 & 3.44 \\
\hline 10 & 208 & 11.02 \\
\hline
\end{tabular}

Source : Enquête Patrimoine INSEE, ménage d'actifs hors indépendants, 5752 ménages pour l'échantillon total, 1887 ménages pour le sous-échantillon risque 
Tableau 4 : corrélation entre pension et instruments

\begin{tabular}{|l|c|c|c|}
\hline \hline & Pension & Instrument 1 & Instrument 2 \\
\hline \hline Pension & 1 & 0.837 & 0.871 \\
Instrument 1 & 0.837 & 1 & 0.989 \\
Instrument 2 & 0.871 & 0.989 & 1 \\
& & & \\
\hline \hline
\end{tabular}

Source : Enquête Patrimoine INSEE, hors indépendants, 5752 ménages.

Note : Instrument 1 , carrière observée et salaire moyen ; Instrument 2 , carrière par génération. 
Tableau 5 : patrimoine net

\begin{tabular}{|c|c|c|c|c|c|c|c|c|c|}
\hline & \multicolumn{3}{|c|}{$r=0,02$} & \multicolumn{3}{|c|}{$r=0,03$} & \multicolumn{3}{|c|}{$r=0,04$} \\
\hline VARIABLES & OLS & IV1 & IV2 & OLS & IV1 & IV2 & OLS & IV1 & IV2 \\
\hline $\begin{array}{l}\text { pension ajustée } \\
\text { revenu total }\end{array}$ & $\begin{array}{c}-0.426^{\star * *} \\
(0.116) \\
0.494^{* * *} \\
(0.052)\end{array}$ & $\begin{array}{c}-0.295^{\star} \\
(0.173) \\
0.451^{\star * *} \\
(0.067)\end{array}$ & $\begin{array}{c}-0.262 \\
(0.166) \\
0.441^{\star * *} \\
(0.066)\end{array}$ & $\begin{array}{c}-0.845^{\star * *} \\
(0.173) \\
0.615^{\star * *} \\
(0.062)\end{array}$ & $\begin{array}{c}-0.693^{\star * *} \\
(0.247) \\
0.575^{\star * *} \\
(0.078)\end{array}$ & $\begin{array}{c}-0.653^{\star \star *} \\
(0.238) \\
0.565^{\star \star \star} \\
(0.077)\end{array}$ & $\begin{array}{c}-1.453^{\star * *} \\
(0.251) \\
0.725^{\star \star *} \\
(0.072)\end{array}$ & $\begin{array}{c}-1.288^{* * *} \\
(0.344) \\
0.691^{* * *} \\
(0.088)\end{array}$ & $\begin{array}{c}-1.240^{* * *} \\
(0.332) \\
0.682^{\star * *} \\
(0.087)\end{array}$ \\
\hline $\begin{array}{l}\text { pension } \\
\text { revenu passé }\end{array}$ & $\begin{array}{c}-0.103^{* * *} \\
(0.024) \\
0.203^{\star * *} \\
(0.016)\end{array}$ & $\begin{array}{c}-0.160^{* * *} \\
(0.047) \\
0.224^{\star * \star} \\
(0.024)\end{array}$ & $\begin{array}{c}-0.146^{* * *} \\
(0.042) \\
0.219^{\star * *} \\
(0.022)\end{array}$ & $\begin{array}{c}-0.154^{\star * *} \\
(0.035) \\
0.187^{\star * *} \\
(0.016)\end{array}$ & $\begin{array}{c}-0.239^{* * *} \\
(0.072) \\
0.211^{* * *} \\
(0.025)\end{array}$ & $\begin{array}{c}-0.211^{\star * *} \\
(0.064) \\
0.203^{* * *} \\
(0.023)\end{array}$ & $\begin{array}{c}-0.216^{\star * *} \\
(0.048) \\
0.170^{\star * *} \\
(0.015)\end{array}$ & $\begin{array}{c}-0.325^{\star \star *} \\
(0.098) \\
0.193^{\star \star \star} \\
(0.025)\end{array}$ & $\begin{array}{c}-0.277^{* * *} \\
(0.088) \\
0.182^{\star * *} \\
(0.023)\end{array}$ \\
\hline
\end{tabular}

Source : Enquête Patrimoine INSEE, hors indépendants, 5752 ménages.

Notes: écart-types robustes entre parenthèses, ${ }^{* * *} p<0.01,{ }^{* *} p<0.05,{ }^{*} p<0.1$

Contrôles : âge, éducation, statut matrimonial, type de famille, héritage. 
Tableau 6 : patrimoine net, sous échantillon risque

\begin{tabular}{|c|c|c|c|c|c|c|c|c|c|}
\hline \multirow[b]{2}{*}{ VARIABLES } & \multicolumn{3}{|c|}{$r=0,02$} & \multicolumn{3}{|c|}{$r=0,03$} & \multicolumn{3}{|c|}{$r=0,04$} \\
\hline & OLS & IV1 & IV2 & OLS & IV1 & IV2 & OLS & IV1 & IV2 \\
\hline $\begin{array}{l}\text { pension ajustée } \\
\text { revenu total }\end{array}$ & $\begin{array}{c}-0.349^{* *} \\
(0.177) \\
0.458^{\star * *} \\
(0.074)\end{array}$ & $\begin{array}{c}-0.330 \\
(0.250) \\
0.452^{\star * *} \\
(0.093)\end{array}$ & $\begin{array}{c}-0.318 \\
(0.233) \\
0.448^{\star * *} \\
(0.087)\end{array}$ & $\begin{array}{c}-0.733^{\star * *} \\
(0.257) \\
0.572^{\star * *} \\
(0.086)\end{array}$ & $\begin{array}{c}-0.714^{* *} \\
(0.348) \\
0.567^{* * *} \\
(0.105)\end{array}$ & $\begin{array}{c}-0.713^{* *} \\
(0.319) \\
0.567^{* * *} \\
(0.097)\end{array}$ & $\begin{array}{c}-1.300^{\star * *} \\
(0.363) \\
0.679^{\star * *} \\
(0.098)\end{array}$ & $\begin{array}{c}-1.278^{* * *} \\
(0.474) \\
0.675^{\star * *} \\
(0.116)\end{array}$ & $\begin{array}{c}-1.295^{\star * *} \\
(0.428) \\
0.678^{\star * *} \\
(0.106)\end{array}$ \\
\hline $\begin{array}{l}\text { pension } \\
\text { revenu passé }\end{array}$ & $\begin{array}{c}-0.100^{* * *} \\
(0.037) \\
0.198^{\star * *} \\
(0.024)\end{array}$ & $\begin{array}{c}-0.196^{\star * *} \\
(0.072) \\
0.236^{\star * *} \\
(0.034)\end{array}$ & $\begin{array}{c}-0.183^{* * *} \\
(0.064) \\
0.231^{\star * *} \\
(0.031)\end{array}$ & $\begin{array}{c}-0.153^{\star * *} \\
(0.055) \\
0.184^{\star * *} \\
(0.023)\end{array}$ & $\begin{array}{c}-0.288^{\star * *} \\
(0.109) \\
0.223^{\star * *} \\
(0.036)\end{array}$ & $\begin{array}{c}-0.267^{\star * *} \\
(0.096) \\
0.216^{* * *} \\
(0.032)\end{array}$ & $\begin{array}{c}-0.219^{* * *} \\
(0.076) \\
0.167^{\star * *} \\
(0.022)\end{array}$ & $\begin{array}{c}-0.377^{\star * *} \\
(0.143) \\
0.201^{\star * \star} \\
(0.034)\end{array}$ & $\begin{array}{c}-0.344^{* * *} \\
(0.126) \\
0.194^{\star * *} \\
(0.030)\end{array}$ \\
\hline
\end{tabular}

Source : Enquête Patrimoine INSEE, hors indépendants, sous-échantillon "risque", 1887 ménages

Notes: écart-types robustes entre parenthèses, ${ }^{* *} p<0.01,{ }^{* *} p<0.05,{ }^{*} p<0.1$

Contrôles : âge, éducation, statut matrimonial, type de famille, héritage. 
Tableau 7 : patrimoine net en fonction de la retraite et de la préférence pour le présent

\begin{tabular}{|c|c|c|c|c|c|c|c|c|c|}
\hline \multirow[b]{2}{*}{ VARIABLES } & \multicolumn{3}{|c|}{$r=0,02$} & \multicolumn{3}{|c|}{$r=0,03$} & \multicolumn{3}{|c|}{$r=0,04$} \\
\hline & OLS & IV1 & IV2 & OLS & IV1 & IV2 & OLS & IV1 & IV2 \\
\hline \multirow[b]{2}{*}{ pension ajustée } & \multicolumn{9}{|c|}{ Forte préférence pour le présent } \\
\hline & $\begin{array}{l}-0.570 \\
(0.413)\end{array}$ & $\begin{array}{l}-0.345 \\
(0.452)\end{array}$ & $\begin{array}{l}-0.389 \\
(0.451)\end{array}$ & $\begin{array}{c}-1.184^{* *} \\
(0.590)\end{array}$ & $\begin{array}{l}-0.831 \\
(0.640)\end{array}$ & $\begin{array}{l}-0.916 \\
(0.631)\end{array}$ & $\begin{array}{c}-2.133^{\star \star \star} \\
(0.820)\end{array}$ & $\begin{array}{l}-1.604^{*} \\
(0889)\end{array}$ & $\begin{array}{c}-1.750^{* *} \\
(0.863)\end{array}$ \\
\hline \multirow[t]{2}{*}{ revenu total } & $0.463^{\star * \star}$ & $0.383^{* *}$ & $0.399^{* *}$ & $0.643^{* * *}$ & $0.541^{* * *}$ & $0.566^{\star \star \star}$ & $0.833^{* * *}$ & $0.708^{* * *}$ & $0.743^{\star \star *}$ \\
\hline & $(0.172)$ & $(0.181)$ & $(0.180)$ & $(0.198)$ & $(0.207)$ & $(0.205)$ & $(0.221)$ & $(0.230)$ & $(0.225)$ \\
\hline \multirow{3}{*}{$\begin{array}{l}\text { pension } \\
\text { revenu passé }\end{array}$} & $\begin{array}{c}-0.206^{\star *} \\
(0.092)\end{array}$ & $\begin{array}{c}-0.276^{\star *} \\
(0.129)\end{array}$ & $\begin{array}{c}-0.269^{\star *} \\
(0.125)\end{array}$ & $\begin{array}{c}-0.325^{\star *} \\
(0.136)\end{array}$ & $\begin{array}{c}-0.422^{* *} \\
(0.197)\end{array}$ & $\begin{array}{c}-0.417^{\star *} \\
(0.192)\end{array}$ & $\begin{array}{c}-0.468^{\star *} \\
(0.184)\end{array}$ & $\begin{array}{c}-0.564^{\star *} \\
(0.262)\end{array}$ & $\begin{array}{c}-0.565^{\star *} \\
(0.255)\end{array}$ \\
\hline & $0.222^{* * *}$ & $0.253^{\star \star \star}$ & $0.250^{\star \star *}$ & $0.223^{\star * *}$ & $0.254^{\star \star *}$ & $0.253^{\star \star \star}$ & $0.216^{\star \star *}$ & $0.239^{* * *}$ & $0.240^{* * *}$ \\
\hline & $(0.053)$ & $(0.065)$ & $(0.063)$ & $(0.054)$ & $(0.070)$ & $(0.068)$ & $(0.052)$ & $(0.068)$ & $(0.066)$ \\
\hline \multirow{3}{*}{ pension ajustée } & \multicolumn{9}{|c|}{ Situation médiane } \\
\hline & -0.166 & 0.026 & -0.003 & -0.422 & -0.166 & -0.220 & -0.843 & -0.505 & -0.597 \\
\hline & $(0.278)$ & $(0.424)$ & $(0.400)$ & $(0.406)$ & $(0.594)$ & $(0.555)$ & $(0.593)$ & $(0.823)$ & $(0.762)$ \\
\hline \multirow[t]{2}{*}{ revenu total } & $0.442^{\star \star *}$ & $0.376^{\star \star}$ & $0.386^{\star \star *}$ & $0.539^{\star \star \star}$ & $0.470^{\star \star \star}$ & $0.485^{\star \star \star}$ & $0.638^{\star \star \star}$ & $0.566^{\star \star \star}$ & $0.585^{\star \star \star}$ \\
\hline & $(0.121)$ & $(0.153)$ & $(0.146)$ & $(0.138)$ & $(0.170)$ & $(0.162)$ & $(0.156)$ & $(0.186)$ & $(0.177)$ \\
\hline \multirow{4}{*}{$\begin{array}{l}\text { pension } \\
\text { revenu passé }\end{array}$} & $-0.098^{*}$ & -0.159 & -0.148 & -0.136 & -0.203 & -0.187 & -0.176 & -0.234 & -0.210 \\
\hline & $(0.059)$ & $(0.141)$ & $(0.124)$ & $(0.085)$ & $(0.204)$ & $(0.178)$ & $(0.114)$ & $(0.256)$ & $(0.223)$ \\
\hline & $0.217^{\star \star *}$ & $0.241^{* * *}$ & $0.237^{\star * *}$ & $0.195^{\star \star *}$ & $0.215^{\star \star \star}$ & $0.210^{\star \star \star}$ & $0.172^{\star \star \star}$ & $0.185^{\star \star *}$ & $0.180^{\star * *}$ \\
\hline & $(0.038)$ & $(0.060)$ & $(0.053)$ & $(0.037)$ & $(0.063)$ & $(0.055)$ & $(0.034)$ & $(0.058)$ & $(0.051)$ \\
\hline \multirow{5}{*}{ pension ajustée } & \multicolumn{9}{|c|}{ Faible préférence pour le présent } \\
\hline & -0.306 & -0.487 & -0.412 & $-0.636^{*}$ & -0.876 & -0.797 & $-1.085^{* *}$ & $-1.375^{\star}$ & $-1.301^{\star \star}$ \\
\hline & $(0.266)$ & $(0.419)$ & $(0.371)$ & $(0.378)$ & $(0.567)$ & $(0.490)$ & $(0.514)$ & $(0.742)$ & $(0.629)$ \\
\hline & $0.446^{\star \star \star}$ & $0.503^{\star \star \star}$ & $0.479^{\star \star \star}$ & $0.539^{\star \star *}$ & $0.600^{\star \star \star}$ & $0.580^{\star \star \star}$ & $0.616^{\star \star \star}$ & $0.675^{\star \star \star}$ & $0.660^{\star \star \star}$ \\
\hline & $(0.111)$ & $(0.151)$ & $(0.135)$ & $(0.131)$ & $(0.168)$ & $(0.149)$ & $(0.148)$ & $(0.184)$ & $(0.161)$ \\
\hline \multirow{4}{*}{$\begin{array}{l}\text { pension } \\
\text { revenu passé }\end{array}$} & -0.070 & $-0.198^{*}$ & $-0.178^{\star}$ & -0.107 & $-0.299^{*}$ & $-0.264^{*}$ & -0.155 & $-0.399^{*}$ & $-0.346^{*}$ \\
\hline & $(0.055)$ & $(0.116)$ & $(0.099)$ & $(0.084)$ & $(0.178)$ & $(0.149)$ & $(0.117)$ & $(0.236)$ & $(0.195)$ \\
\hline & $0.186^{\star \star \star}$ & $0.232^{\star \star *}$ & $0.225^{\star \star \star}$ & $0.168^{\star \star *}$ & $0.220^{* * *}$ & $0.210^{\star \star \star}$ & $0.151^{\star \star \star}$ & $0.200^{\star \star *}$ & $0.189^{\star \star \star}$ \\
\hline & $(0.037)$ & $(0.053)$ & $(0.047)$ & $(0.036)$ & $(0.056)$ & $(0.048)$ & $(0.034)$ & $(0.054)$ & $(0.046)$ \\
\hline
\end{tabular}

Source : Enquête Patrimoine INSEE, hors indépendants, 5752 ménages.

Notes: écart-types robustes entre parenthèses, ${ }^{* * *} p<0.01,{ }^{* *} p<0.05,{ }^{*} p<0.1$ 
Tableau 8: Effet de substitution pour les différentes composantes du patrimoine, $r=0.03$

\begin{tabular}{|c|c|c|c|c|c|c|c|}
\hline \multirow{2}{*}{ VARIABLES } & \multirow{2}{*}{ Patrimoine net } & \multicolumn{2}{|c|}{ Immobilier } & \multicolumn{4}{|c|}{ Financier } \\
\hline & & Total & autre immobilier & Total & épargne retraite & assurance vie & valeurs mobilières \\
\hline & \multicolumn{7}{|c|}{ "Sans instrumentation } \\
\hline \multirow[t]{2}{*}{ pension ajustée } & $-0.845^{\star \star *}$ & $-0.406^{\star *}$ & -0.355 & $-0.333^{* * *}$ & $-0.103^{* * *}$ & $-0.110^{*}$ & -0.175 \\
\hline & $(0.173)$ & $(0.196)$ & $(0.290)$ & $(0.068)$ & $(0.038)$ & $(0.066)$ & $(0.131)$ \\
\hline \multirow[t]{2}{*}{ revenu total } & $0.615^{\star * *}$ & $0.450^{* * *}$ & $0.268^{* * *}$ & $0.170^{\star * *}$ & $0.043^{* * *}$ & $0.086^{\star * *}$ & $0.200^{* * *}$ \\
\hline & $(0.062)$ & $(0.061)$ & $(0.081)$ & $(0.026)$ & $(0.011)$ & $(0.021)$ & $(0.056)$ \\
\hline \multirow[t]{2}{*}{ pension } & $-0.154^{\star * \star}$ & 0.036 & 0.021 & $-0.063^{\star * \star}$ & 0.008 & -0.021 & -0.015 \\
\hline & $(0.035)$ & $(0.046)$ & $(0.076)$ & $(0.014)$ & $(0.008)$ & $(0.016)$ & $(0.036)$ \\
\hline \multirow[t]{3}{*}{ revenu passé } & $0.187^{\star \star *}$ & $0.124^{\star \star \star}$ & $0.063^{* *}$ & $0.048^{\star \star \star}$ & 0.005 & $0.030^{\star \star \star}$ & $0.067^{\star \star *}$ \\
\hline & $(0.016)$ & $(0.018)$ & $(0.026)$ & $(0.006)$ & $(0.003)$ & $(0.007)$ & $(0.019)$ \\
\hline & & & & IV 1 & & & \\
\hline \multirow[t]{2}{*}{ pension ajustée } & $-0.693^{\star \star \star}$ & -0.495 & -0.284 & $-0.384^{\star * *}$ & $-0.134^{\star *}$ & -0.108 & -0.192 \\
\hline & $(0.247)$ & $(0.313)$ & $(0.491)$ & $(0.087)$ & $(0.064)$ & $(0.119)$ & $(0.268)$ \\
\hline \multirow[t]{2}{*}{ revenu total } & $0.575^{\star * *}$ & $0.472^{\star * *}$ & $0.251^{*}$ & $0.184^{\star * *}$ & $0.051^{* * *}$ & $0.086^{\star *}$ & $0.204^{* *}$ \\
\hline & $(0.078)$ & $(0.089)$ & $(0.132)$ & $(0.029)$ & $(0.017)$ & $(0.033)$ & $(0.079)$ \\
\hline \multirow[t]{2}{*}{ pension } & $-0.239^{* * *}$ & -0.014 & 0.074 & $-0.118^{* * *}$ & -0.009 & -0.054 & -0.070 \\
\hline & $(0.072)$ & $(0.099)$ & $(0.162)$ & $(0.024)$ & $(0.019)$ & $(0.038)$ & $(0.093)$ \\
\hline \multirow[t]{3}{*}{ revenu passé } & $0.211^{* * *}$ & $0.138^{* \star *}$ & 0.048 & $0.064^{\star * \star}$ & $0.009^{*}$ & $0.039^{* * *}$ & $0.083^{* * *}$ \\
\hline & $(0.025)$ & $(0.031)$ & $(0.049)$ & $(0.009)$ & $(0.006)$ & $(0.012)$ & $(0.031)$ \\
\hline & & & & IV 2 & & & \\
\hline \multirow[t]{2}{*}{ pension ajustée } & $-0.653^{\star * *}$ & $-0.584^{*}$ & -0.285 & $-0.338^{\star * *}$ & $-0.139^{\star *}$ & -0.103 & -0.305 \\
\hline & $(0.238)$ & $(0.301)$ & $(0.470)$ & $(0.079)$ & $(0.061)$ & $(0.112)$ & $(0.256)$ \\
\hline \multirow[t]{2}{*}{ revenu total } & $0.565^{\star \star *}$ & $0.495^{\star \star *}$ & $0.251^{* *}$ & $0.172^{\star \star *}$ & $0.052^{* * *}$ & $0.084^{\star * *}$ & $0.233^{\star * *}$ \\
\hline & $(0.077)$ & $(0.087)$ & $(0.127)$ & $(0.026)$ & $(0.017)$ & $(0.032)$ & $(0.080)$ \\
\hline \multirow[t]{2}{*}{ pension } & $-0.211^{* * *}$ & -0.027 & 0.068 & $-0.097^{* * *}$ & -0.007 & -0.043 & -0.101 \\
\hline & $(0.064)$ & $(0.089)$ & $(0.146)$ & $(0.021)$ & $(0.016)$ & (0.034) & $(0.084)$ \\
\hline \multirow[t]{2}{*}{ revenu passé } & $0.203^{* * *}$ & $0.142^{* * *}$ & 0.050 & $0.058^{* * *}$ & $0.009^{*}$ & $0.036^{\star * *}$ & $0.092^{* * *}$ \\
\hline & $(0.023)$ & $(0.028)$ & $(0.045)$ & $(0.008)$ & $(0.005)$ & $(0.011)$ & $(0.030)$ \\
\hline
\end{tabular}

Source : Enquête Patrimoine INSEE, hors indépendants, 5752 ménages.

Notes: écart-types robustes entre parenthèses, ${ }^{* *} p<0.01,{ }^{* *} p<0.05,{ }^{*} p<0.1, r=0,03$ et $x=-0,03$ 
Tableau 9 : Effets marginaux immobilier et épargne retraite

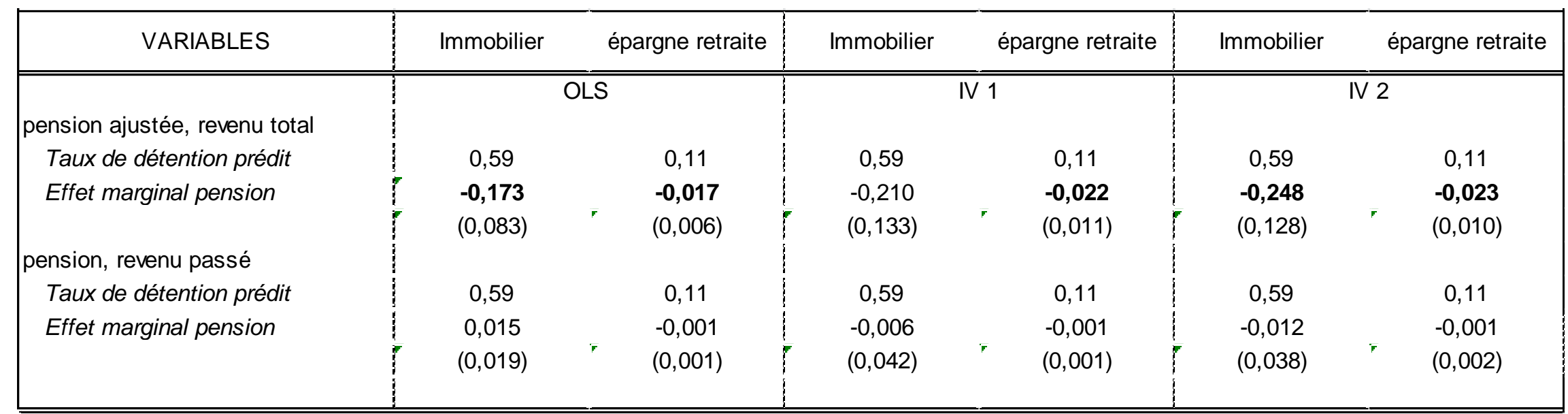

Source : Enquête Patrimoine INSEE, hors indépendants, 5752 ménages.

Note : $r=0,03$ et $x=-0,03$ 
Graphique 1 : distribution des âges de la personne de référence

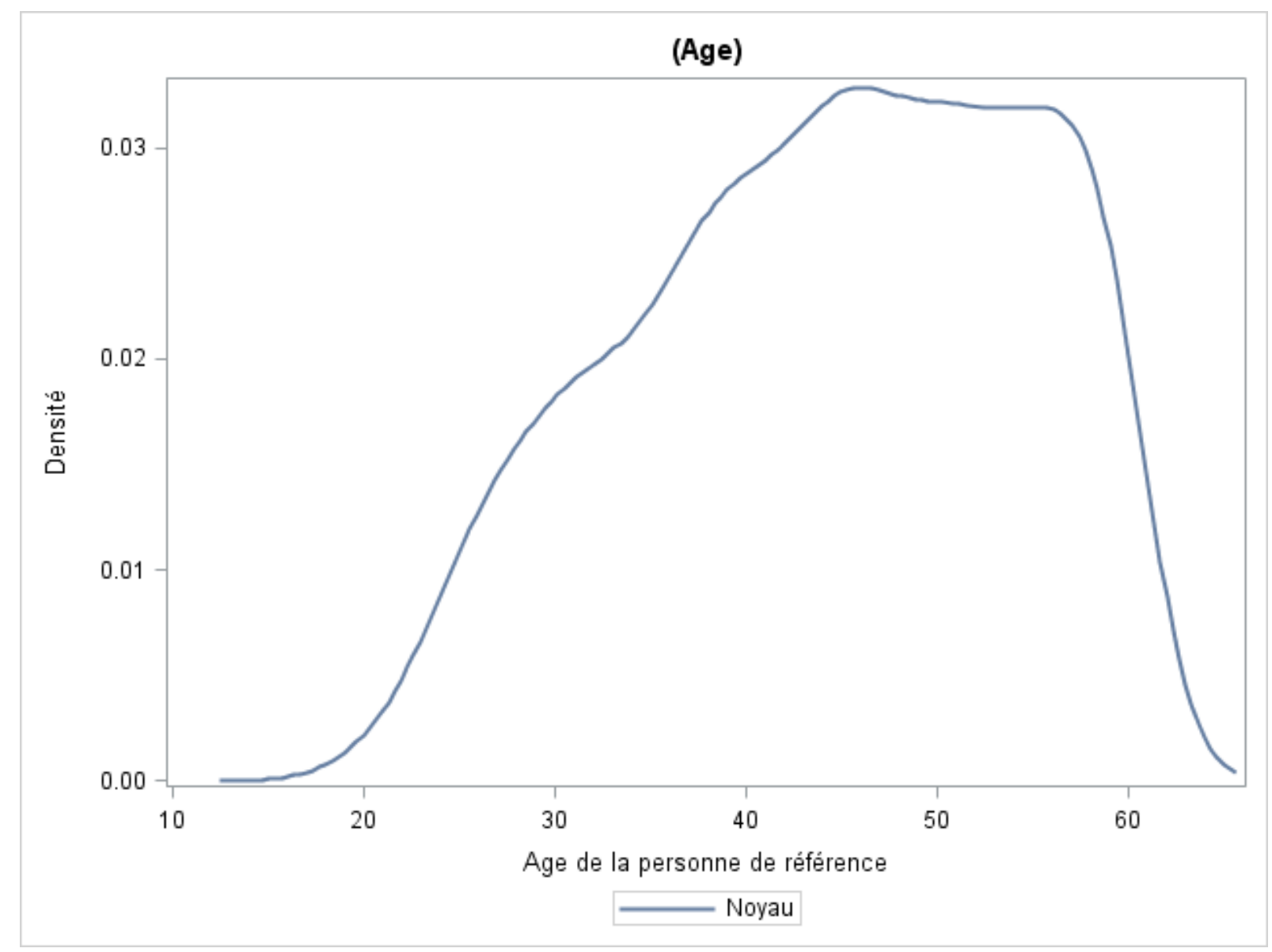

Source : Enquête Patrimoine INSEE,

hors indépendants, 5752 ménages 
Graphiqe 2 : distribution du $Q$ de Gale en function de $r$
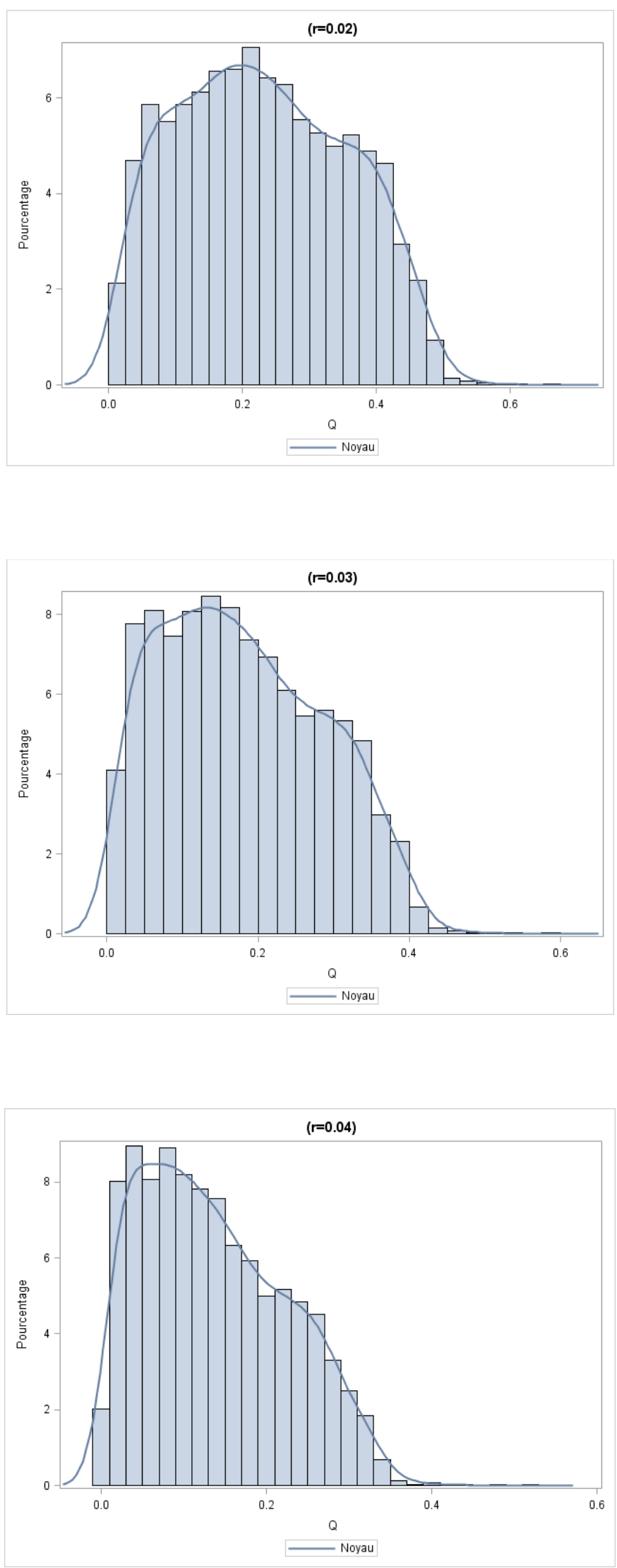

Source : Enquête Patrimoine INSEE, hors indépendants, 5752 ménages 
Graphique 3 : distributions des revenus, en fonction de $r$
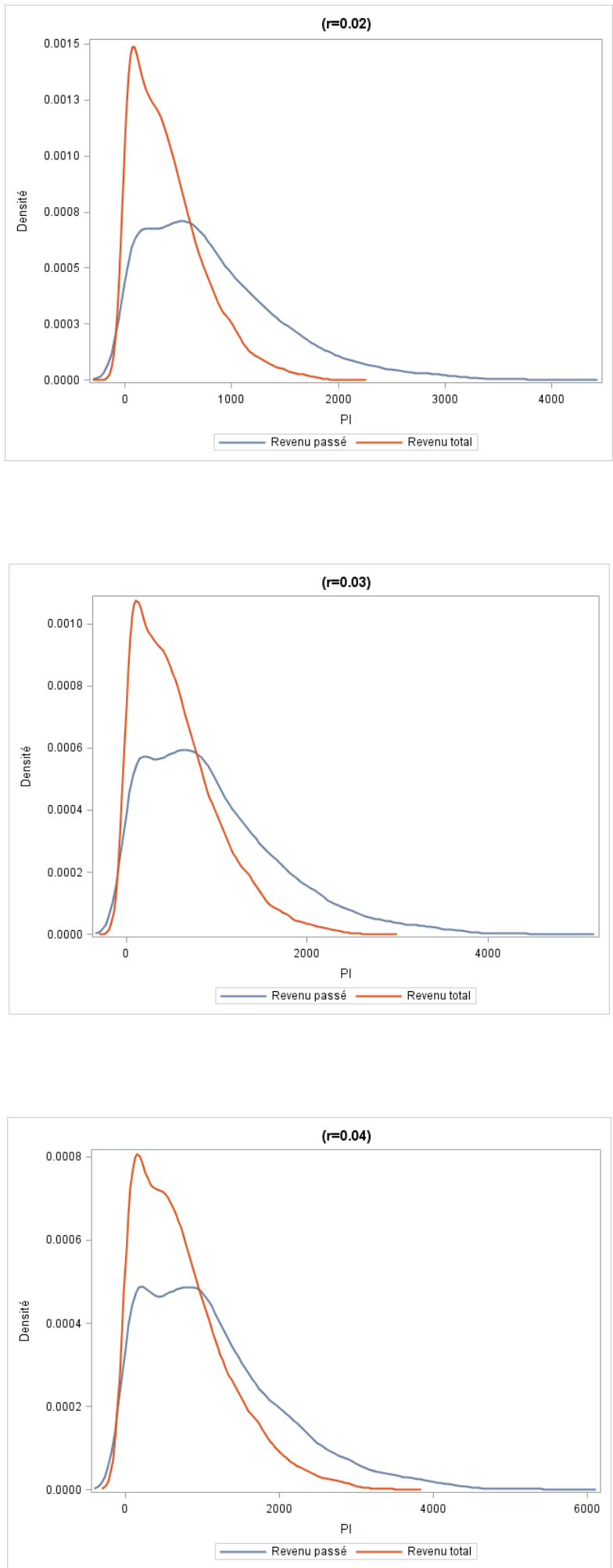

Source : Enquête Patrimoine INSEE, hors indépendants, 5752 ménages 
Graphique 4 : distributions des pensions, en fonction de $r$
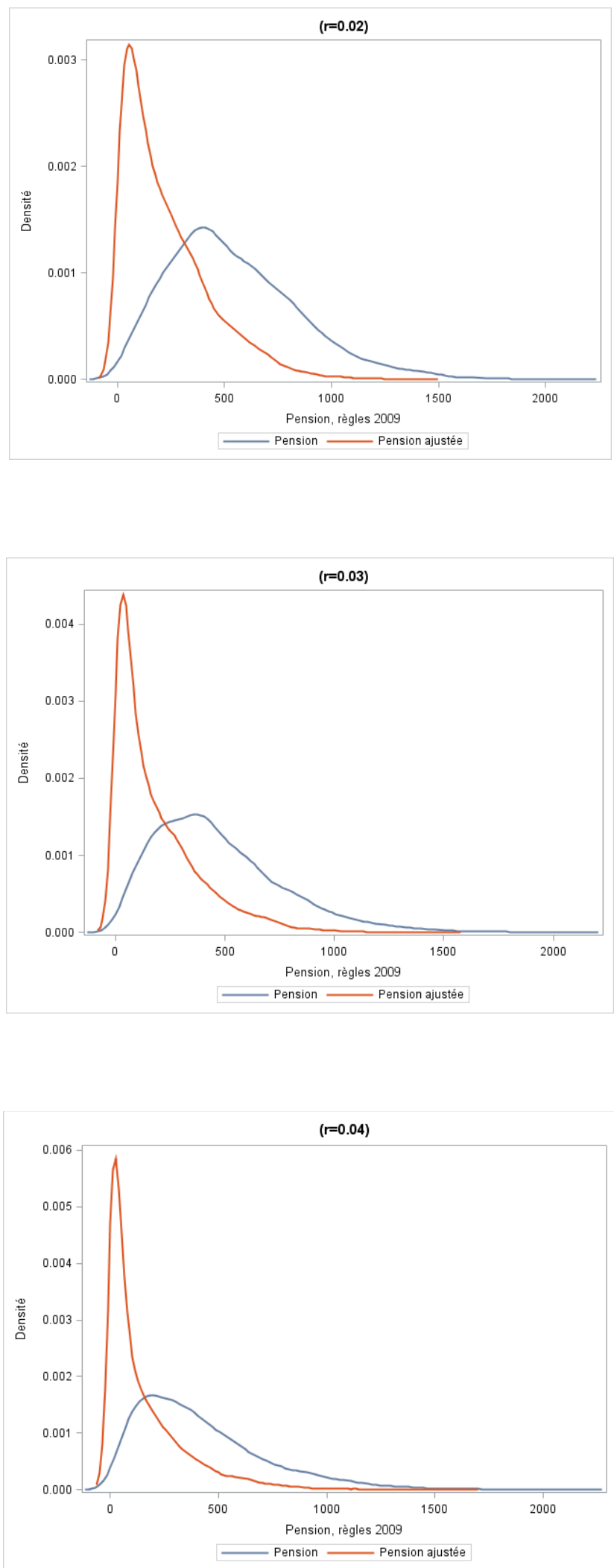

Source : Enquête Patrimoine INSEE, hors indépendants, 5752 ménages 


\section{Annexe}

Tableau A1 : variables sociodémographiques

\begin{tabular}{|c|c|c|}
\hline & Fréquence & Pourcentage \\
\hline \multicolumn{3}{|l|}{ Type de ménage } \\
\hline Personne seule & 1202 & 20.90 \\
\hline Famille monoparentale & 757 & 13.16 \\
\hline Couple sans enfant & 1110 & 19.30 \\
\hline Couple avec enfants & 2502 & 43.50 \\
\hline Autre type de ménage (ménage complexe) & 181 & 3.15 \\
\hline \multicolumn{3}{|l|}{ Type de famille } \\
\hline Conjoint actif sans enfant & 594 & 10.33 \\
\hline Conjoint actif, 1 enfant & 622 & 10.81 \\
\hline Conjoint actif, 2 enfants & 718 & 12.48 \\
\hline Conjoint actif, 3 enfants ou plus & 312 & 5.42 \\
\hline Conjoint inactif sans enfant & 540 & 9.39 \\
\hline Conjoint inactif, 1 enfant & 299 & 5.20 \\
\hline Conjoint inactif, 2 enfants & 320 & 5.56 \\
\hline Conjoint inactif, 3 enfants ou plus & 280 & 4.87 \\
\hline Famille monoparentale / père & 159 & 2.76 \\
\hline Famille monoparentale / mère & 598 & 10.40 \\
\hline Homme seul & 634 & 11.02 \\
\hline Femme seule & 568 & 9.87 \\
\hline Autres cas & 108 & 1.88 \\
\hline \multicolumn{3}{|l|}{ Statut Matrimonial } \\
\hline Célibataire & 2070 & 35.99 \\
\hline Marié(e) ou remarié(e), y compris séparé(e) légalement & 2832 & 49.24 \\
\hline Veuf(ve) & 158 & 2.75 \\
\hline Divorcé(e) & 692 & 12.03 \\
\hline \multicolumn{3}{|l|}{ Education } \\
\hline Certificat d'études primaires ou moins & 1083 & 18.83 \\
\hline Brevet des collèges & 345 & 6.00 \\
\hline BEP/CAP & 1621 & 28.18 \\
\hline Baccalauréat & 861 & 14.97 \\
\hline $\mathrm{Bac}+2$ & 617 & 10.73 \\
\hline Master (université) & 531 & 9.23 \\
\hline Master (grande école) & 272 & 4.73 \\
\hline Diplôme de 3e cycle & 422 & 7.34 \\
\hline
\end{tabular}

Source : Enquête Patrimoine INSEE, ménage d'actifs hors indépendants, 5752 ménages 
Tableau A2 : Effet de substitution pour les différentes composantes du patrimoine, $r=0.02$

\begin{tabular}{|c|c|c|c|c|c|c|c|}
\hline \multirow{2}{*}{ VARIABLES } & \multirow{2}{*}{ Patrimoine net } & \multicolumn{2}{|c|}{ Immobilier } & \multicolumn{4}{|c|}{ Financier } \\
\hline & & Total & autre immobilier & Total & épargne retraite & assurance vie & valeurs mobilières \\
\hline & \multicolumn{7}{|c|}{ Sans instrumentation } \\
\hline \multirow[t]{2}{*}{ pension ajustée } & $-0.426^{\star * \star}$ & -0.175 & -0.177 & $-0.202^{\star \star \star}$ & $-0.065^{\star *}$ & -0.053 & -0.070 \\
\hline & $(0.116)$ & $(0.137)$ & $(0.205)$ & $(0.046)$ & $(0.025)$ & $(0.046)$ & $(0.090)$ \\
\hline revenu total & $0.494^{\star * \star}$ & $0.380^{* * *}$ & $0.219^{\star * *}$ & $0.141^{\star \star \star}$ & $0.038^{\star \star \star}$ & $0.071^{\star * *}$ & $0.167^{\star \star \star}$ \\
\hline \multirow[t]{3}{*}{ revenu passé } & $0.203^{\star \star \star}$ & $0.139^{* * *}$ & $0.066^{\star *}$ & $0.050^{\star * *}$ & $0.005^{\star}$ & $0.032^{* \star *}$ & $0.074^{* * *}$ \\
\hline & $(0.016)$ & $(0.019)$ & $(0.028)$ & $(0.007)$ & $(0.003)$ & $(0.007)$ & $(0.020)$ \\
\hline & & & & IV 1 & & & \\
\hline \multirow[t]{2}{*}{ pension ajustée } & $-0.295^{\star}$ & -0.238 & -0.164 & $-0.238^{\star * \star}$ & $-0.085^{\star}$ & -0.060 & -0.091 \\
\hline & $(0.173)$ & $(0.227)$ & $(0.359)$ & $(0.061)$ & $(0.045)$ & $(0.086)$ & $(0.198)$ \\
\hline \multirow[t]{2}{*}{ revenu total } & $0.451^{\star * \star}$ & $0.400^{* * *}$ & $0.215^{\star}$ & $0.152^{* * *}$ & $0.044^{\star * \star}$ & $0.073^{\star *}$ & $0.174^{\star *}$ \\
\hline & $(0.067)$ & $(0.080)$ & $(0.120)$ & $(0.025)$ & $(0.015)$ & $(0.030)$ & $(0.071)$ \\
\hline \multirow[t]{2}{*}{ pension } & $-0.160^{\star * *}$ & 0.020 & 0.098 & $-0.073^{\star * \star}$ & -0.000 & -0.038 & -0.041 \\
\hline & $(0.047)$ & $(0.068)$ & $(0.113)$ & $(0.016)$ & $(0.012)$ & $(0.026)$ & $(0.065)$ \\
\hline \multirow[t]{3}{*}{ revenu passé } & $0.224^{\star * \star}$ & $0.147^{\star \star *}$ & 0.043 & $0.063^{* * *}$ & 0.008 & $0.041^{* * *}$ & $0.087^{\star * \star}$ \\
\hline & $(0.024)$ & $(0.030)$ & $(0.046)$ & $(0.008)$ & $(0.005)$ & $(0.011)$ & $(0.030)$ \\
\hline & & & & IV 2 & & & \\
\hline \multirow[t]{2}{*}{ pension ajustée } & -0.262 & -0.290 & -0.171 & $-0.205^{\star \star \star}$ & $-0.089^{\star *}$ & -0.052 & -0.166 \\
\hline & $(0.166)$ & $(0.218)$ & $(0.345)$ & $(0.056)$ & $(0.042)$ & $(0.081)$ & (0.189) \\
\hline \multirow[t]{2}{*}{ revenu total } & $0.441^{* * *}$ & $0.417^{* * *}$ & $0.217^{\star}$ & $0.142^{\star \star *}$ & $0.045^{\star \star \star}$ & $0.070^{\star *}$ & $0.198^{* * *}$ \\
\hline & $(0.066)$ & $(0.078)$ & $(0.116)$ & $(0.023)$ & $(0.015)$ & $(0.028)$ & $(0.071)$ \\
\hline \multirow[t]{2}{*}{ pension } & $-0.146^{\star * *}$ & 0.010 & 0.086 & $-0.062^{\star * \star}$ & 0.001 & -0.030 & -0.061 \\
\hline & $(0.042)$ & $(0.061)$ & $(0.102)$ & $(0.014)$ & $(0.011)$ & $(0.023)$ & $(0.059)$ \\
\hline \multirow[t]{2}{*}{ revenu passé } & $0.219^{* * *}$ & $0.151^{* * *}$ & 0.047 & $0.059^{\star * *}$ & $0.008^{\star}$ & $0.039^{\star * *}$ & $0.095^{\star * *}$ \\
\hline & $(0.022)$ & $(0.027)$ & $(0.043)$ & $(0.008)$ & $(0.005)$ & $(0.010)$ & $(0.029)$ \\
\hline
\end{tabular}

Source : Enquête Patrimoine INSEE, hors indépendants, 5752 ménages.

Notes: écart-types robustes entre parenthèses, ${ }^{* * *} p<0.01,{ }^{* *} p<0.05,{ }^{*} p<0.1, r=0,02$ et $x=-0,02$ 
Tableau A3 : Effet de substitution pour les différentes composantes du patrimoine, $r=0.04$

\begin{tabular}{|c|c|c|c|c|c|c|c|}
\hline \multirow{2}{*}{ VARIABLES } & \multirow{2}{*}{ Patrimoine net } & \multicolumn{2}{|c|}{ Immobilier } & \multicolumn{4}{|c|}{ Financier } \\
\hline & & Total & autre immobilier & Total & épargne retraite & assurance vie & valeurs mobilières \\
\hline & \multicolumn{7}{|c|}{ Sans instrumentation } \\
\hline \multirow[t]{2}{*}{ pension ajustée } & $-1.453^{\star \star *}$ & $-0.734^{* * *}$ & -0.613 & $-0.510^{\star \star *}$ & $-0.153^{\star \star *}$ & $-0.188^{* *}$ & $-0.329^{*}$ \\
\hline & $(0.251)$ & $(0.271)$ & $(0.398)$ & $(0.098)$ & $(0.056)$ & $(0.093)$ & $(0.188)$ \\
\hline \multirow[t]{2}{*}{ revenu total } & $0.725^{\star \star \star}$ & $0.510^{\star \star \star}$ & $0.315^{\star \star \star}$ & $0.196^{\star \star \star}$ & $0.047^{\star \star \star}$ & $0.100^{\star \star \star}$ & $0.229^{\star \star \star}$ \\
\hline & $(0.072)$ & $(0.067)$ & $(0.089)$ & $(0.029)$ & $(0.013)$ & $(0.024)$ & $(0.065)$ \\
\hline \multirow[t]{2}{*}{ pension } & $-0.216^{\star * *}$ & 0.013 & -0.015 & $-0.089^{* * *}$ & 0.003 & -0.032 & -0.032 \\
\hline & $(0.048)$ & $(0.060)$ & $(0.096)$ & $(0.020)$ & $(0.010)$ & $(0.021)$ & $(0.044)$ \\
\hline \multirow[t]{3}{*}{ revenu passé } & $0.170^{\star \star \star}$ & $0.112^{* * \star}$ & $0.063^{\star \star \star}$ & $0.045^{\star \star \star}$ & $0.005^{\star}$ & $0.027^{\star * \star}$ & $0.061^{* * *}$ \\
\hline & $(0.015)$ & $(0.016)$ & $(0.024)$ & $(0.006)$ & $(0.003)$ & $(0.006)$ & $(0.017)$ \\
\hline & & & & IV 1 & & & \\
\hline \multirow[t]{2}{*}{ pension ajustée } & $-1.288^{\star \star \star}$ & $-0.850^{\star *}$ & -0.443 & $-0.580^{* * *}$ & $-0.200^{* *}$ & -0.173 & -0.339 \\
\hline & $(0.344)$ & $(0.421)$ & $(0.654)$ & $(0.122)$ & $(0.091)$ & $(0.162)$ & $(0.358)$ \\
\hline \multirow[t]{2}{*}{ revenu total } & $0.691^{* * *}$ & $0.534^{\star \star \star}$ & $0.281^{* *}$ & $0.211^{\star * *}$ & $0.057^{\star * \star}$ & $0.096^{\star * *}$ & $0.231^{\star * *}$ \\
\hline & $(0.088)$ & $(0.097)$ & $(0.141)$ & $(0.032)$ & $(0.020)$ & $(0.036)$ & $(0.087)$ \\
\hline \multirow[t]{2}{*}{ pension } & $-0.325^{\star \star \star}$ & -0.080 & 0.017 & $-0.166^{* * *}$ & -0.023 & -0.070 & -0.107 \\
\hline & $(0.098)$ & $(0.130)$ & $(0.211)$ & $(0.033)$ & $(0.025)$ & $(0.050)$ & $(0.121)$ \\
\hline \multirow[t]{3}{*}{ revenu passé } & $0.193^{* * *}$ & $0.132^{* * *}$ & 0.056 & $0.061^{* * *}$ & $0.011^{*}$ & $0.035^{\star * *}$ & $0.077^{* * *}$ \\
\hline & $(0.025)$ & $(0.030)$ & $(0.047)$ & $(0.008)$ & $(0.006)$ & $(0.012)$ & $(0.029)$ \\
\hline & & & & IV 2 & & & \\
\hline \multirow[t]{2}{*}{ pension ajustée } & $-1.240^{\star \star *}$ & $-0.988^{\star *}$ & -0.430 & $-0.516^{* * *}$ & $-0.206^{* *}$ & -0.176 & -0.502 \\
\hline & $(0.332)$ & $(0.406)$ & $(0.625)$ & $(0.110)$ & $(0.086)$ & $(0.151)$ & $(0.341)$ \\
\hline \multirow[t]{2}{*}{ revenu total } & $0.682^{* * *}$ & $0.562^{\star \star \star}$ & $0.278^{\star *}$ & $0.197^{\star \star \star}$ & $0.058^{\star \star *}$ & $0.097^{\star * \star}$ & $0.265^{\star \star \star}$ \\
\hline & $(0.087)$ & $(0.094)$ & $(0.136)$ & $(0.030)$ & $(0.019)$ & $(0.034)$ & $(0.087)$ \\
\hline \multirow[t]{2}{*}{ pension } & $-0.277^{\star \star \star}$ & -0.093 & 0.026 & $-0.134^{* * *}$ & -0.020 & -0.055 & -0.150 \\
\hline & $(0.088)$ & $(0.118)$ & $(0.191)$ & $(0.028)$ & $(0.022)$ & $(0.044)$ & $(0.109)$ \\
\hline \multirow[t]{2}{*}{ revenu passé } & $0.182^{\star \star *}$ & $0.135^{\star * *}$ & 0.054 & $0.054^{* * *}$ & $0.010^{* *}$ & $0.032^{\star * *}$ & $0.086^{* * *}$ \\
\hline & $(0.023)$ & $(0.027)$ & $(0.043)$ & $(0.007)$ & $(0.005)$ & $(0.010)$ & $(0.028)$ \\
\hline
\end{tabular}

Source : Enquête Patrimoine INSEE, hors indépendants, 5752 ménages.

Notes: écart-types robustes entre parenthèses, ${ }^{* *} p<0.01,{ }^{* *} p<0.05,{ }^{*} p<0.1, r=0,04$ et $x=-0,04$ 